\title{
Business Analytics and IT in Smart Grid - Part 2: The Qualitative Mitigation Impact of Piecewise Monotonic Data Approximations on the iSHM Class Map Footprints of Overhead Low-Voltage Broadband over Power Lines Topologies Contaminated by Measurement Differences
}

\author{
Athanasios G. Lazaropoulos ${ }^{1,2, *}$ \\ 1: School of Electrical and Computer Engineering / National Technical University of Athens / \\ 9 Iroon Polytechniou Street / Zografou, GR 15780 \\ 2: Department of Industrial Design and Production Engineering / School of Engineering / University of \\ West Attica / 250 Thivon \& P. Ralli / Athens, GR 12244
}

Received March 25, 2020; Accepted May 4, 2020; Published May 13, 2020

\begin{abstract}
Business analytics and IT infrastructure preserve the integrity of the smart grid (SG) operation against the flood of big data that may be susceptible to faults, such as measurement differences. In [1], the impact of measurement differences that follow continuous uniform distributions (CUDs) of different magnitudes has been investigated via initial Statistical Hybrid Model (iSHM) footprints during the operation of overhead low-voltage broadband over power lines (OV LV BPL) networks. In this companion paper, the mitigation efficiency of piecewise monotonic data approximations, such as L1PMA and L2WPMA, is qualitatively assessed in terms of iSHM footprints when the aforementioned measurement difference CUD of different intensities are applied.
\end{abstract}

Keywords: Smart Grid; Broadband over Power Lines (BPL) networks; Power Line Communications (PLC); Distribution and Transmission Power Grids; Capacity, Statistics; Business Analytics; IT; Modeling 


\section{Nomenclature}

\begin{tabular}{|l|l|}
\hline BPL & Broadband over Power Lines \\
\hline BPMN & Business Process Model and Notation \\
\hline CASD & $\begin{array}{l}\text { Channel Attenuation Statistical } \\
\text { Distribution }\end{array}$ \\
\hline CUD & Continuous Uniform Distribution \\
deterministic hybrid model \\
\hline DHM & $\begin{array}{l}\text { Fault and Instability Identification } \\
\text { Methodology }\end{array}$ \\
\hline FIM & hook style energy theft detection method \\
\hline HS-DET method & Internet Protocol \\
\hline IP & Information Technology \\
\hline IT & initial Statistical Hybrid Model \\
\hline iSHM & Line-of-Sight \\
\hline LOS & Low Voltage \\
\hline LV & L1 Piecewise Monotonic Approximation \\
\hline L1PMA & $\begin{array}{l}\text { L2 Weighted Piecewise Monotonic } \\
\text { Approximation }\end{array}$ \\
\hline L2WPMA & Maximum Likelihood Estimator \\
\hline MLE & Normal Distribution \\
\hline ND & Overhead \\
\hline OV & Percent Error Sum \\
\hline PES & Smart Grid \\
\hline SG & Statistical Hybrid Model \\
\hline SHM & Topology Identification Methodology \\
\hline TIM & Wire-to-Ground \\
\hline WtG & \\
\hline
\end{tabular}




\section{Introduction}

During the recent years, the transformation of the traditional power grid to the SG urges the installation of a parallel advanced IP-based communications network enhanced with a plethora of broadband applications and business analytics [1]-[14]. Among the available communications solutions that can support this communications network, BPL networks can play an important role since they exploit the already installed wired power grid infrastructure [4], [15]-[23].

However, big data that overwhelm SG are susceptible to errors that can affect business analytics and decisions based on them. Indeed, as the operation of BPL networks is concerned across SG, the already installed wired power grid infrastructure is a hostile medium for communications as it is designed to deliver power rather information [19], [21], [24]-[27]. As the BPL channel modeling is concerned, the recently proposed iSHM, which is based on the well-validated DHM, can be deployed for the broadband channel description of transmission and distribution power grids [19]-[23], [28]-[31]. Also, a plethora of related broadband iSHM tools, such as the definition procedure, the class maps and the iSHM footprints, have been so far demonstrated and tested in order to assist the operation of iSHM towards a more accurate statistical description of the communications channel [32]-[35]. Except for the communications channel itself, measurement differences between the experimental and theoretical results during the channel attenuation determination, briefly denoted as measurement differences, may occur due to a number of practical reasons and "real-life" difficulties that may critically influence iSHM operation, the interaction of broadband iSHM tools with iSHM and finally the SG big data with the related decisions.

Actually, the impact of measurement differences that can be treated as CUDs has been assessed through the iSHM footprints for a list of real indicative OV LV BPL topologies in [1] while appropriate countermeasures that are based on piecewise monotonic data approximations and iSHM footprints are first presented and assessed in this companion paper. The numerical results of [1] confirmed the nasty impact of measurement differences on the behavior of iSHM footprints of the OV LV BPL topologies since high measurement differences may jam the SG broadband tools, such as the topology identification technique and energy theft identification, which are based on the analysis of iSHM footprints. To restore the affected iSHM footprints and to preserve the quality of business analytics, piecewise monotonic data approximations, such as L1PMA [36] and L2WPMA [37], which have been successfully applied in distribution and transmission BPL networks for the mitigation of measurement differences in broadband applications of TIM [8], FIIM [8] and HS-DET method [38], can also be applied in iSHM footprints so that the contaminated measured data can be partially restored and the approximated iSHM footprints tend to be gathered close to the respective theoretical values.

The rest of this paper is organized as follows: Section II synopsizes the mathematics of measurement differences. Also, the piecewise monotonic data approximations of interest, say L1PMA and L2WPMA, are briefly outlined. L1PMA and L2WPMA mitigation character is analyzed in mathematical terms as well as their involvement in the iSHM operation and iSHM footprints. In Section III, the numerical results regarding the mitigation impact of piecewise monotonic data approximations against the measurement differences on the iSHM footprints are shown. Section IV concludes this paper. 


\section{Measurement Differences and Countermeasures in DHM and iSHM Footprints}

In accordance with [1], measurement differences affect the iSHM performance since they are mathematically superimposed on the numerical results of DHM, which is the core element of the Phase A of the BPMN diagram of iSHM [29]. First, a synopsis of the mathematical involvement of the CUD measurement differences in DHM is provided. Second, a presentation of the two piecewise monotonic data approximations of interest, say L1PMA and L2WPMA, is presented as well as the required mathematics.

\subsection{Measurement Differences in DHM and iSHM Footprint Operation Settings}

Due to practical reasons and "real-life" conditions [36], [39], [40], measurement differences may be observed among the theoretical and measured coupling scheme transfer functions for given OV LV BPL topology and coupling scheme. In accordance with [1], [36], [39], [40], these measurement differences can be decently treated either as CUDs of variable maximum value $a_{\text {CUD }}$ or as NDs of variable standard deviation $\sigma_{\mathrm{ND}}$ when the mean value $\mu_{\mathrm{ND}}$ is assumed to be equal to zero. Since measurement differences $e_{d 1, d 2, i}^{D}\{\cdot\}$ that follow the aforementioned distributions are added to the theoretical coupling scheme transfer function $H^{\mathrm{OVLV}, C}\{\cdot\}$, the measured coupling scheme transfer function $\overline{H_{d 1, d 2, l}^{\mathrm{OVLV}, D}}\{\cdot\}$ can be determined by [1], [6], [41]

$$
\overline{H_{d 1, d 2, l}^{\mathrm{OVLV}, D}}\left(f_{q}\right)=H^{\mathrm{OVLV}, C}\left(f_{q}\right)+e_{d 1, d 2, i}^{D}\left(f_{q}\right), q=1, \ldots, Q, i=1, \ldots, I
$$

where $[\cdot]^{C}$ denotes the applied coupling scheme, $[\cdot]^{D}$ denotes the applied measurement difference distribution -either CUD of this paper or ND-, $f_{q}$ is the flat-fading subchannel start frequency, $Q$ is the number of subchannels in the examined frequency range, $d 1$ is the first parameter of the applied measurement difference distribution (i.e., the minimum value $-a_{\text {CUD }}$ of CUD), $d 2$ is the second parameter of the applied measurement difference distribution (i.e., the maximum value $a_{\mathrm{CUD}}$ of CUD), $H^{\mathrm{OVLV}, \mathrm{C}}\left(f_{q}\right)$ is the theoretical coupling scheme transfer function at frequency $f_{q}$ for given coupling scheme, $e_{d 1, d 2, i}^{D}\left(f_{q}\right)$ is the measurement difference at frequency $f_{q}$ for given measurement difference distribution, $I$ is the number of different $1 \times Q$ line vectors of measurement differences per applied measurement difference distribution, first and second parameter and $i$ indicates the $i^{\text {th }}$ among $I$ line vectors of measurement differences.

\subsection{Piecewise Monotonic Data Approximations and iSHM Footprints}

Piecewise monotonic data approximations have extensively been applied in transmission and distribution BPL topologies during various critical broadband applications that require the mitigation of measurement differences in order to ensure the smooth operation of the power grid [36], [39], [42]-[46]. A synthesis of suitable quantitative performance metrics has been proposed and benchmarked so far, such as PES, fault PES and $\triangle \mathrm{PES}$, for the mitigation of measurement differences during the BPL channel attenuation determination [44], [14]. During the preparation of the improved iSHM footprints, piecewise monotonic data approximations are going to filter the numerical results of DHM that are contaminated by measurement differences between the Phases A and B of the BPMN diagram of iSHM [29]. From the available piecewise monotonic data approximations [37], [42], [47]-[51], L1PMA and L2WPMA are applied 
in this paper while the corresponding iSHM footprints can be considered as new qualitative performance metrics.

As L1PMA is integrated in BPMN diagram of iSHM, L1PMA receives as input the measured OV LV BPL coupling scheme transfer function data from Phase A and gives as output the corresponding approximated data that are further delivered to Phase $\mathrm{B}$ of the BPMN diagram of iSHM. By exploiting the piecewise monotonicity property of OV LV BPL coupling scheme transfer functions, L1PMA decomposes the input data into separate monotonous sections between the adjacent turning points (primary extrema) [49], [50]. The main advantage of L1PMA is its mitigation performance against the uncorrelated measurement differences generated by CUDs by identifying and ignoring the few large measurement differences [36], [42], [44]. In programming terms, by having developed and exploiting the appropriate MATLAB - Octave / Fortran interface, L1PMA Fortran software package, which is freely available in [52], gives the best fit of the measured OV LV BPL coupling scheme transfer function data given the number of monotonic sections (i.e., either user- or computer-defined).

As L2WPMA is regarded, L2WPMA holds the same position with L1PMA in the BPMN diagram of iSHM. Similarly to L1PMA, appropriate Fortran software package that interoperates with the existing MATLAB - Octave module is freely available online in [37]. L2WPMA operates in a same way to L1PMA since L2WPMA decomposes the examined input measured data contaminated by measurement differences into separate monotonous sections between its primary extrema [37], [42], [44]. Conversely to L1PMA, L2WPMA exploits the first divided of input data while it minimizes the weighted sum of the square of the measurement differences by requiring specific number of sign changes that are defined either by user or the computer.

Note that the default operation settings, which are described in Sec 3.4 of [24] and Sec. 3.2 of [1], that regulate the interconnected operation from DHM to iSHM footprints are also assumed in this paper. In order to allow the application of piecewise monotonic data approximations, the only mandatory change concerning the assumed default operation settings has to do with the required BPL frequency range; due to restrictions in the number of monotonic sections and sign changes imposed by the Fortran software packages of L1PMA and L2WPMA, respectively, the BPL frequency range and flat-fading subchannel frequency spacing are assumed to be equal to $3-30 \mathrm{MHz}$ and $1 \mathrm{MHz}$, respectively. Therefore, the number of flat-fading subchannels $Q$ is equal to 27 while the flat-fading subchannel start frequencies are given by

$$
f_{q}=3 \mathrm{MHz}+(q-1) \times 1 \mathrm{MHz}, q=1, \ldots, Q
$$

Note that small differences are expected to appear in iSHM class maps and iSHM footprints of OV LV BPL topologies of [1] due to the aforementioned changes of BPL frequency range settings but the generality of the mitigation measurement difference analysis remains valid.

As the mathematics of piecewise monotonic data approximations is considered, the approximated coupling scheme transfer function can be expressed as

$$
\overline{\overline{H_{d 1, d 2, l}^{\mathrm{OVLV}, C, P}}}\left(f_{q}\right)=P\left\{\overline{H_{d 1, d 2, l}^{\mathrm{OVLV}, C,}}\left(f_{q}\right)\right\}, q=1, \ldots, Q, i=1, \ldots, I
$$

where $[\cdot]^{P}$ denotes the applied piecewise monotonic data approximation, say L1PMA and L2WPMA, and $P\{\cdot\}$ synopsizes the aforementioned procedure for given piecewise monotonic data approximation that is anyway executed by the corresponding software package. 


\section{Numerical Results and Discussion}

In this Section, numerical results that qualitatively assesses the mitigation impact of piecewise monotonic data approximations against measurement differences on iSHM footprints of OV LV BPL topologies are demonstrated. First, the countermeasures effect of L1PMA and L2WPMA is qualitatively benchmarked for given intensity of the measurement difference CUD. Second, the impact of the user-defined numbers of L1PMA monotonic sections and L2WPMA sign changes is graphically assessed with respect to the mitigation of measurement differences. Third, the mitigation performance of L1PMA and L2WPMA is finally qualitatively assessed against the measurement differences of increasing intensity.

\section{1 iSHM Class Maps of OV LV BPL Topologies}

In accordance with the BPMN diagram of iSHM [29], the CASD MLEs of iSHM are computed at the Phase $\mathrm{C}$ of Fig. 2(a) of [24]. In accordance with [35], Weibull CASD MLEs are going to be used in this paper since Weibull CASD performs the best performance among the available iSHM CASDs with reference to the percentage change and average absolute percentage change when OV LV BPL topology main subclasses are examined. In accordance with [33]-[35], the iSHM class map of OV LV BPL topologies, which acts as the graphical basis for the demonstration of iSHM footprints due to

measurement differences, is plotted in Fig. 1 with respect to $\hat{a}_{\mathrm{MLE}}^{\mathrm{Weibull}}, \hat{\beta}_{\mathrm{MLE}}^{\mathrm{Weibull}}$ and the average capacity of each OV LV BPL topology subclass when the default operation settings of [1], [24] and the modified BPL frequency range settings of Sec.2.2 are assumed.

By comparing Fig. 1 with Fig. 1 of [1], differences are observed in capacity borders and the location of the real indicative OV LV BPL topologies of Table 1 of [24] since different frequency range properties from those of [1] are assumed in this paper in order to allow the fine operation of L1PMA and L2WPMA [44]. Also, to focus on the demonstration of the mitigation impact results of piecewise monotonic data approximations, only one real indicative OV LV BPL topology of the main subclasses of Table 1 of [24], say, urban case A, is going to be examined in this paper. According to [1], aggravated OV LV BPL topologies, such as urban case A and B, that are characterized by intense multipath environments, are more sensitive to measurement differences as unveiled in iSHM footprints of [1] and for that reason urban case A is arbitrarily chosen to be investigated in this paper. Note that the real indicative OV LV BPL rural case is located outside the $\hat{\beta}_{\mathrm{MLE}}^{\text {Weibull }}$ upper limit of Fig. 1 due to the aforementioned frequency range properties.

3.2 iSHM Footprints due to Measurement Differences and the Countermeasures of Piecewise Monotonic Data Approximations

The impact of measurement differences on iSHM class maps of OV LV BPL topologies can be examined via the iSHM footprints as well as the effect of the proposed countermeasures. The mitigation impact of piecewise monotonic approximations can be checked by the comparison of the iSHM footprint due to measurement differences and the iSHM footprint after the application of the countermeasures in terms of the footprint size reduction, direction to the axes origin and mitigation shift. 


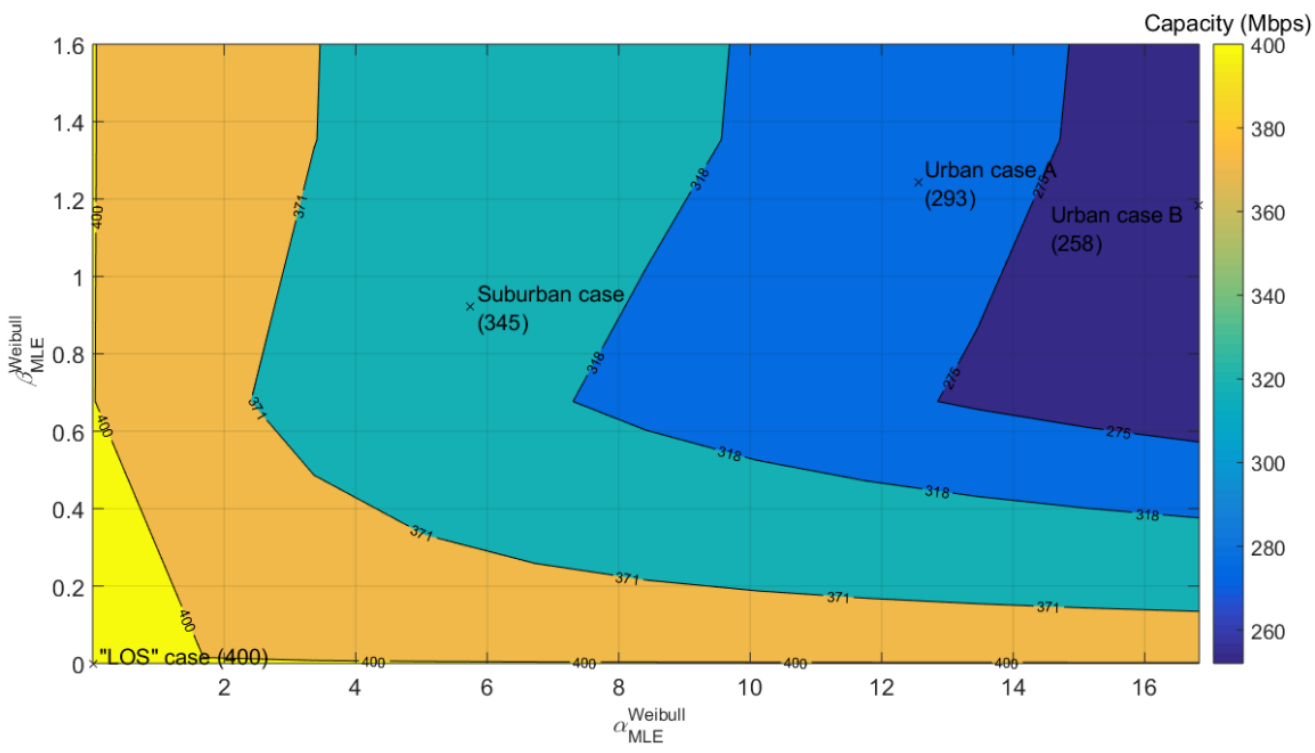

Fig. 1. iSHM class map of OV LV BPL topologies when $3-30 \mathrm{MHz}$ frequency band, $1 \mathrm{MHz}$ frequency subchannel spacing, $\mathrm{WtG}^{1}$ coupling scheme and FCC Part 15 are assumed [35].

With reference to the iSHM class map of Fig. 1, the iSHM footprint due to measurement differences of the arbitrary $5 \mathrm{~dB}$ maximum value $a_{\text {CUD }}$ for the real indicative OV LV BPL urban case A is illustrated in Fig. 2 as superimposed white circles on the iSHM class map. Also, in Fig. 2, the iSHM footprint after the application of L1PMA against the aforementioned measurement differences is shown as superimposed cyan squares when 4 monotonic sections are assumed. Note that 100 line vectors of random measurement differences of the aforementioned CUD are assumed during the preparation of Fig. 2 that imply 100 white circles and 100 respective cyan squares. In Fig. 3, similar iSHM footprints with Fig. 2 are illustrated but for the application of L2WPMA when 4 sign changes and the same 100 line vectors of measurement differences are assumed.

By comparing iSHM footprints due to measurement differences of Figs 2 and 3 with Fig. 3(a) of [1], it is obvious that the fewer data of the numerical results of DHM of this paper render the iSHM footprint due to measurements more sensitive to the measurement differences and more segmented. The destructive result of measurement differences is justified by the extent of the iSHM footprint due to measurement differences that starts from the neighborhood of the theoretical values of $\hat{a}_{\mathrm{MLE}}^{\mathrm{Weibull}}$ and $\hat{\beta}_{\text {MLE }}^{\text {Weibull }}$ of the real indicative OV LV BPL urban case A and reaches up to the OV LV BPL rural class topology.

As the L1PMA is applied, its mitigation efficiency against the measurement differences is visible by the shift of the iSHM footprint towards the up right direction due to the L1PMA application near to the theoretical values of $\hat{a}_{\mathrm{MLE}}^{\mathrm{Weibull}}$ and $\hat{\beta}_{\mathrm{MLE}}^{\mathrm{Weibull}}$ of the real indicative OV LV BPL urban case A. Indeed, cyan squares that come from the L1PMA application are located closer to the theoretical values of $\hat{a}_{\mathrm{MLE}}^{\mathrm{Weibull}}$ and $\hat{\beta}_{\mathrm{MLE}}^{\mathrm{Weibull}}$ of the real indicative OV LV BPL urban case A compared with the white circles supported 
by the measurement differences. In Fig. 3, same results regarding the iSHM footprint of the approximated data are observed after the L2WPMA application.

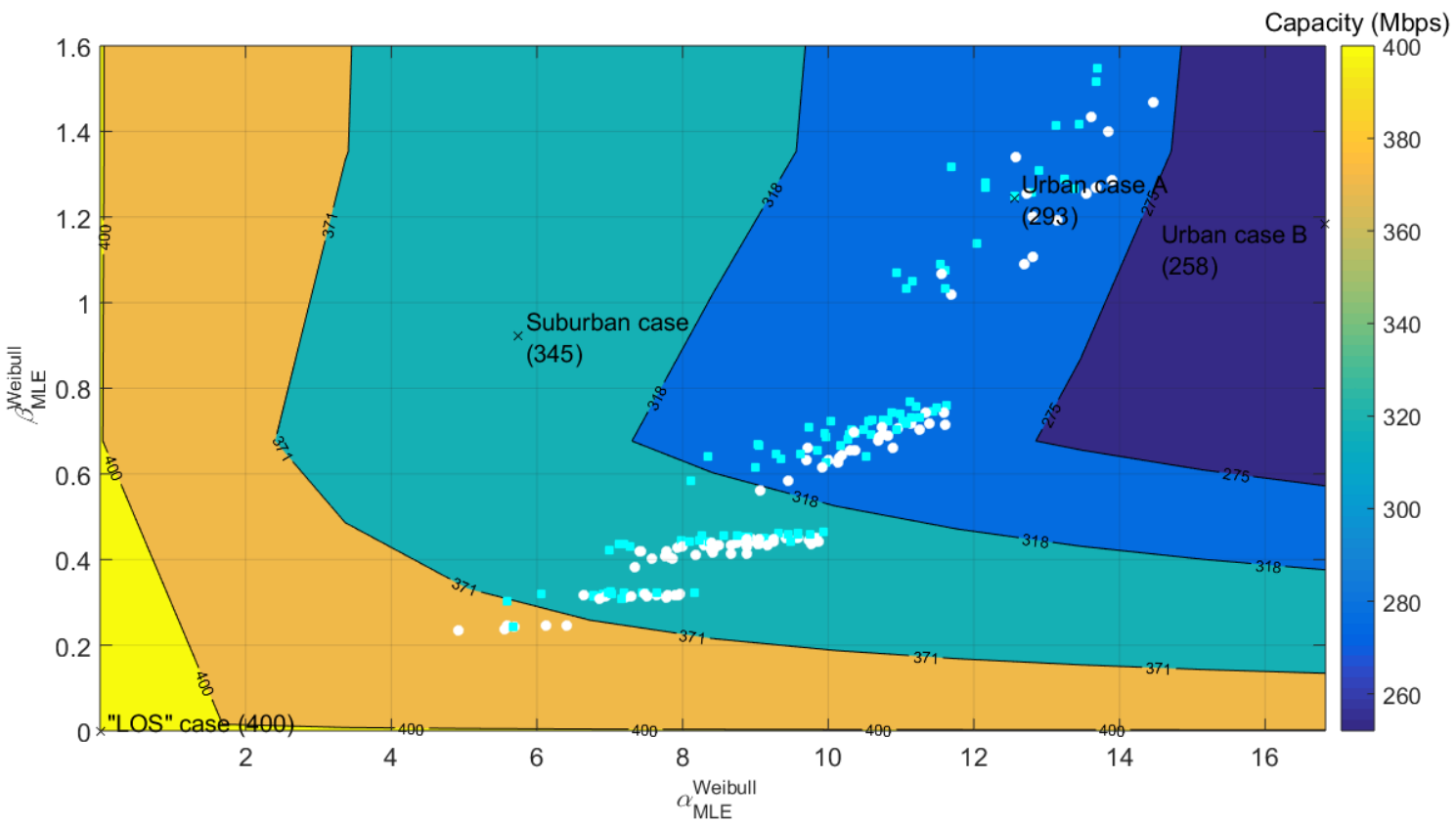

Fig. 2. iSHM footprints of the real indicative OV LV BPL urban case A when 3-30MHz frequency band, $1 \mathrm{MHz}$ frequency subchannel spacing, $\mathrm{WtG}^{1}$ coupling scheme, FCC Part 15, CUD measurement differences of maximum value $a_{\mathrm{CUD}}=5 \mathrm{~dB}$ (white circles) are assumed and L1PMA of 4 monotonic sections (cyan squares) is applied.

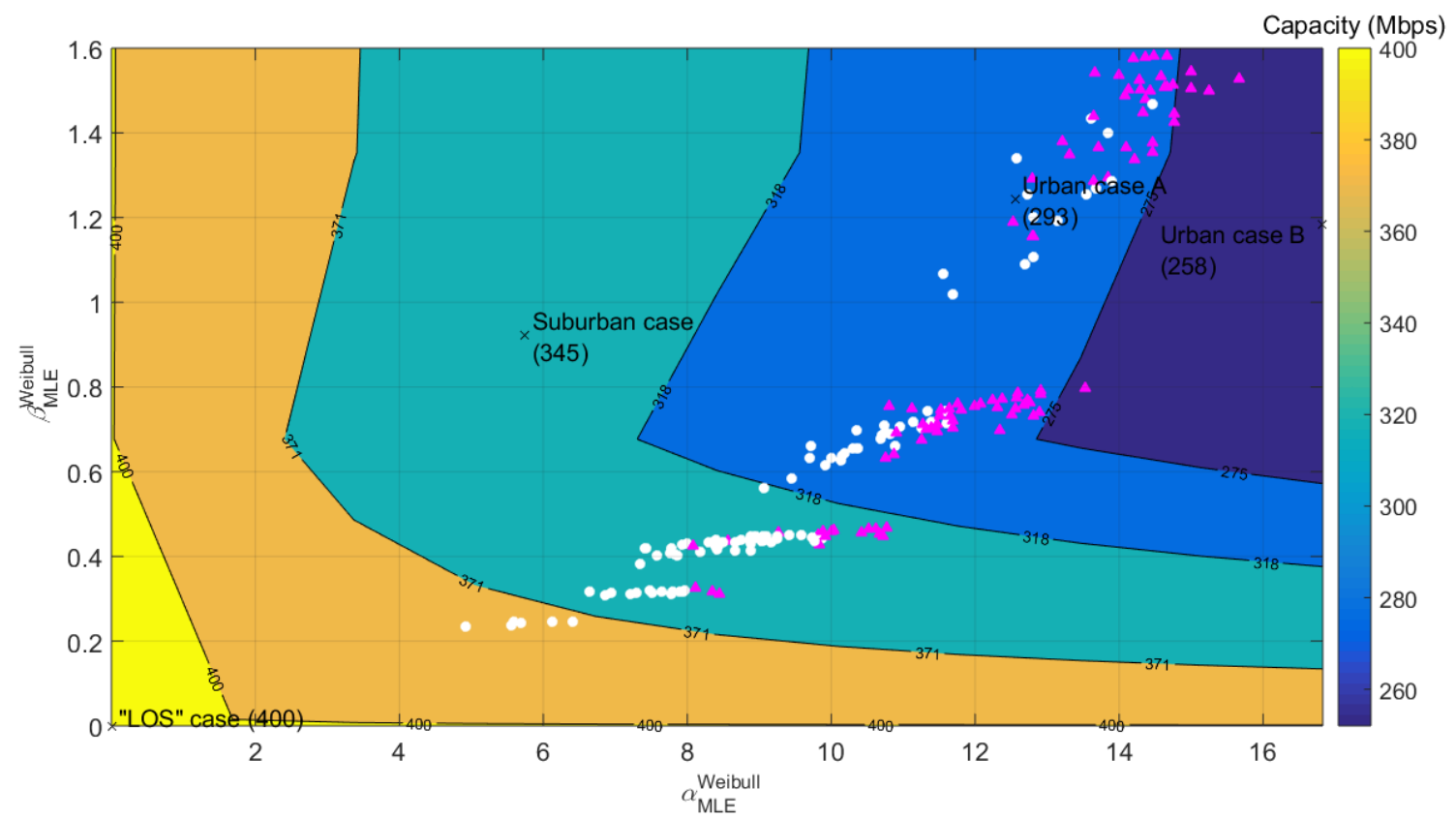

Fig. 3. Same plot with Fig. 2 but for L2WPMA of 4 sign changes (magenta triangles).

Until now, the mitigation impact of measurement differences on class maps has been investigated in terms of the relative location and the extent of the corresponding iSHM footprints due to the application of L1PMA and L2WPMA. In the following 
subsection, the impact of monotonic sections and sign changes of L1PMA and L2WPMA, respectively, is assessed against the measurement differences for given measurement difference intensity.

\subsection{The Role of Monotonic Sections and Sign Changes against the Measurement Differences}

In accordance with [42], [44], the selection of the numbers of monotonic sections for L1PMA and of the sign changes for L2WPMA has a critical effect during their application and their mitigation effect against the measurement differences. In order to highlight the importance of the right selection of the number of L1PMA monotonic sections and of L2WPMA sign changes, various strategies have been applied that exploit either the deterministic definition or the adaptive one until now [36], [39], [42]-[44].

Similarly to Fig. 2, the iSHM footprint due to measurement differences of the arbitrary $6 \mathrm{~dB}$ maximum value $a_{\mathrm{CUD}}$ for the real indicative OV LV BPL urban case A is illustrated in Fig. 4(a). Also, in Fig. 4(a), the iSHM footprint due to the application of L1PMA against the aforementioned measurement differences is shown as superimposed cyan squares when 1 monotonic section is assumed. In Figs. 4(b)-(i), similar footprints with Fig. 4(a) are illustrated but for the number of monotonic sections ranging from 2 to 9, respectively. In Figs. 5(a)-(i), similar footprints with Figs. 4(a)-(i) are illustrated but for the application of L2WPMA when sign changes range from 1 to 9, respectively, and the same 100 line vectors of measurement differences are assumed in Figs. 4(a)-(i) and 5(a)-(i).

From Figs. 4(a)-(i) and 5(a)-(i), it is obvious that the careful selection of L1PMA monotonic sections and L2WPMA sign changes may have different impact on the mitigation of measurement differences whereas a bad selection may even make the approximated data worse than the measured ones. Indeed, as the L1PMA monotonic sections are concerned, the predefined number of monotonic sections forces L1PMA to create a pattern of specific monotonic sections for the approximated coupling scheme transfer function data by appropriately filtering the examined measured coupling scheme transfer function data. On the basis of the predefined number of monotonic sections, the L1PMA concept is that measurement differences that mainly disrupt the pattern for the examined measured coupling scheme transfer function data are ignored thus delivering the approximated coupling scheme transfer function data for given number of monotonic sections. A relatively high number of monotonic sections, which is significantly greater than the number of monotonic sections of the theoretical coupling scheme channel attenuation data (e.g., greater than 8 monotonic sections in Fig. 4), can have the opposite results to the expected ones due to the overapproximation of the measured data; say, in this case, a significant number of contaminated data by measurement differences should be taken into account so that the number of monotonic sections of the approximated coupling scheme transfer function data agrees with the predefined number of monotonic sections. The overapproximation of the measured data, which comes from the application of relatively high numbers of monotonic sections, can be observed in iSHM footprints in the cases where cyan squares of the approximated coupling scheme transfer function data start to coincide with the white circles of the measured coupling scheme transfer function data. Conversely, when significantly lower number of monotonic sections is assumed (e.g., 1 or 2 monotonic sections in Fig. 4), an average value of the measured coupling scheme transfer function data is expected. 


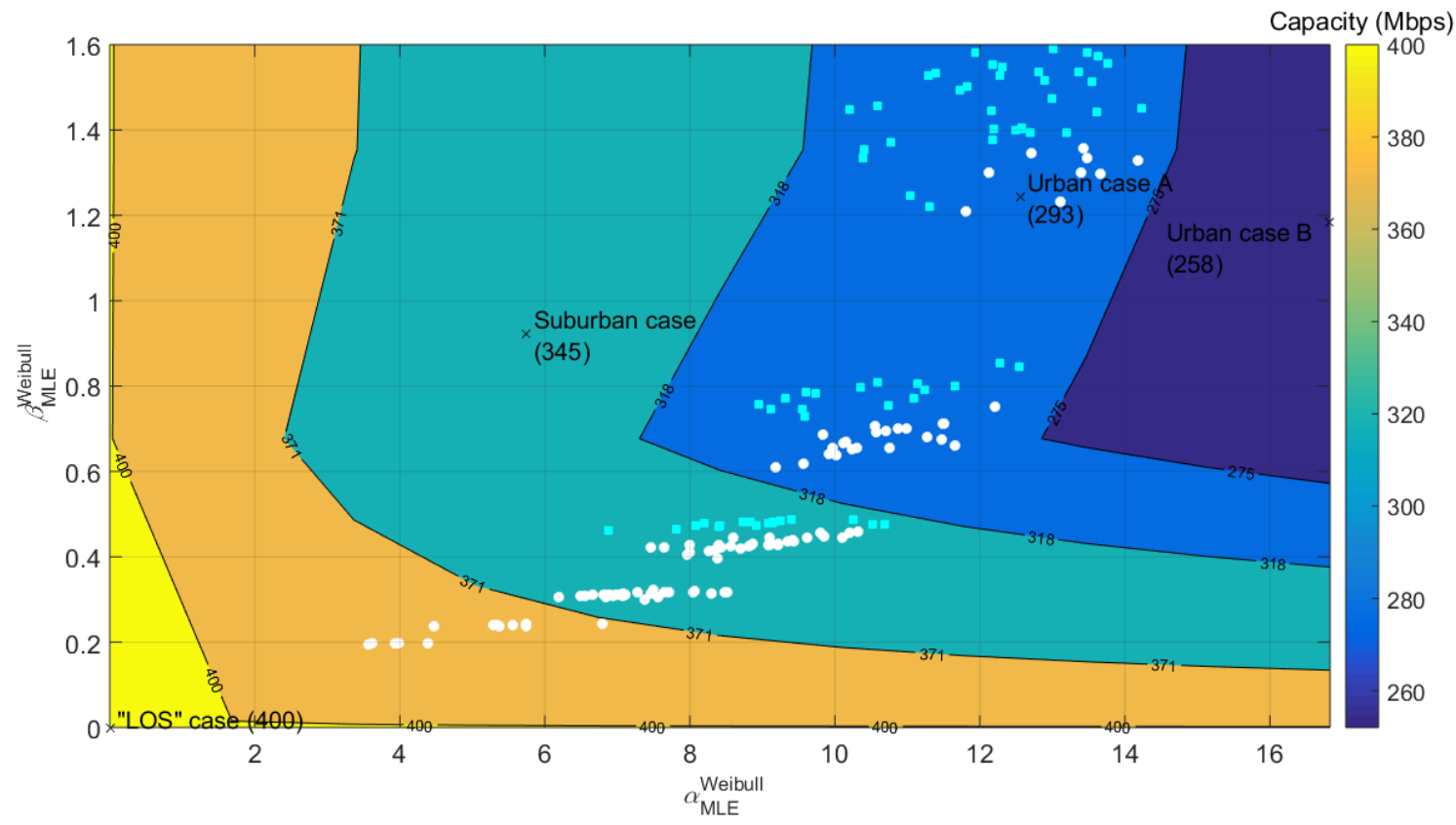

(a)

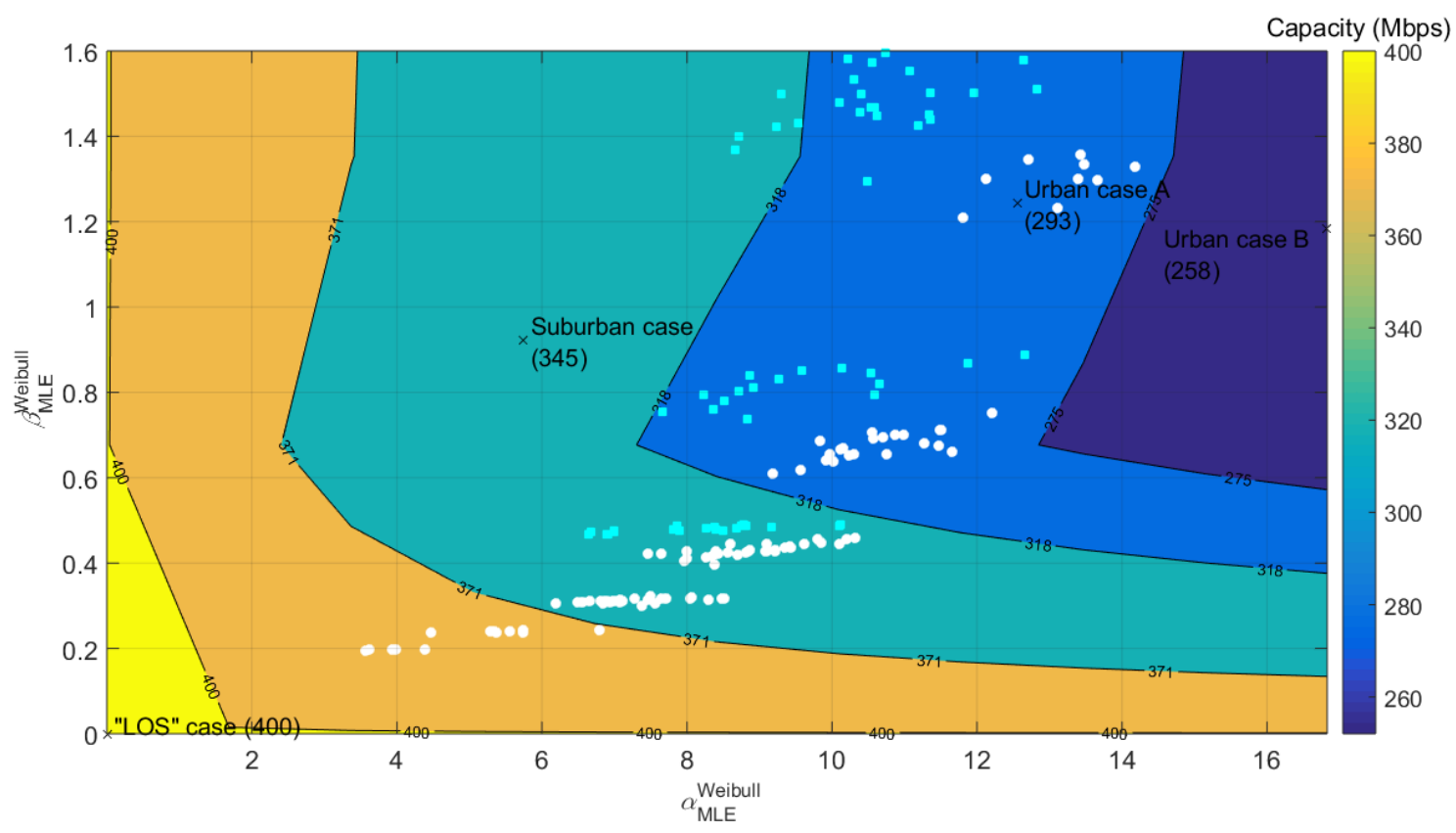

(b) 


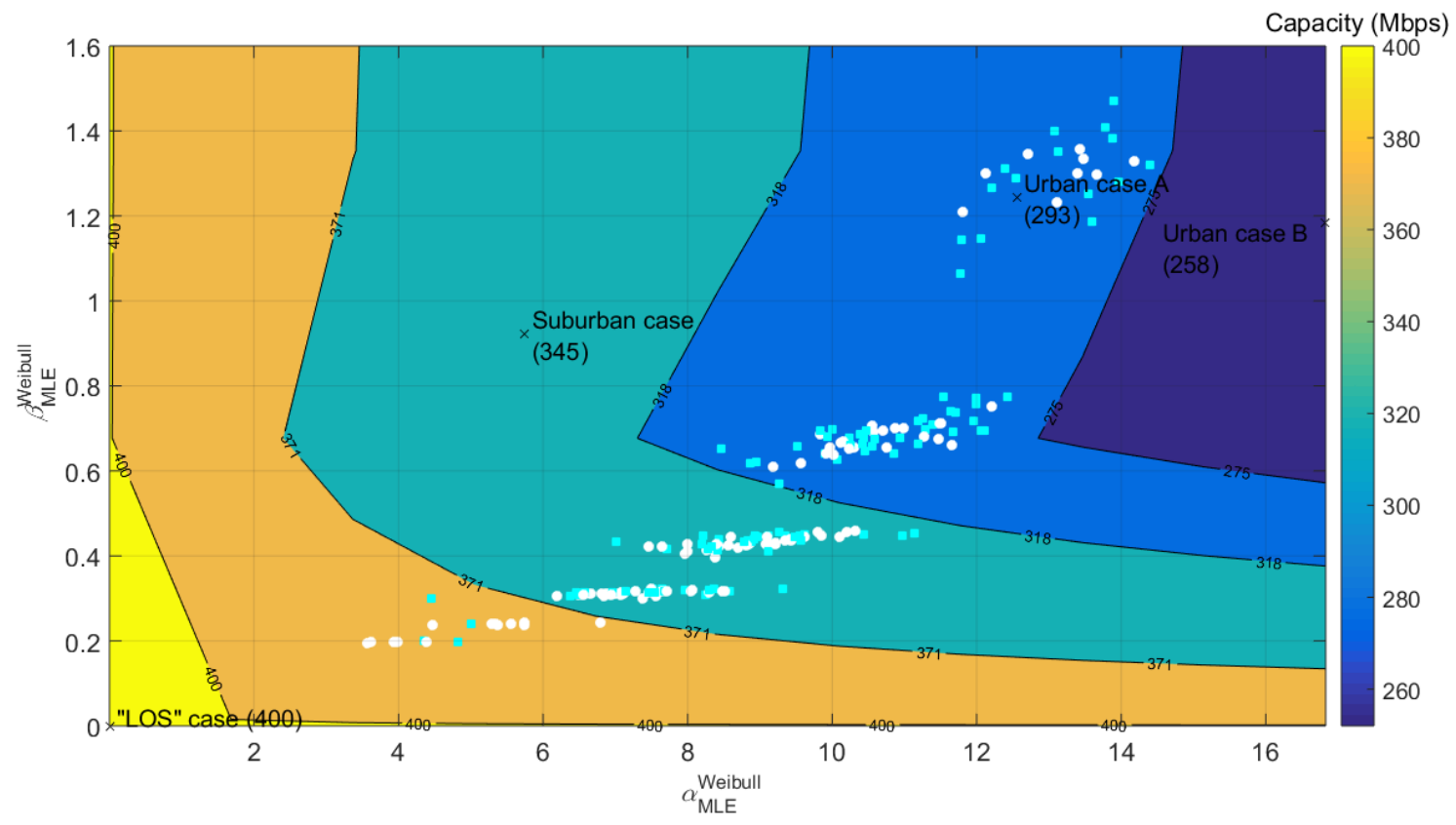

(c)

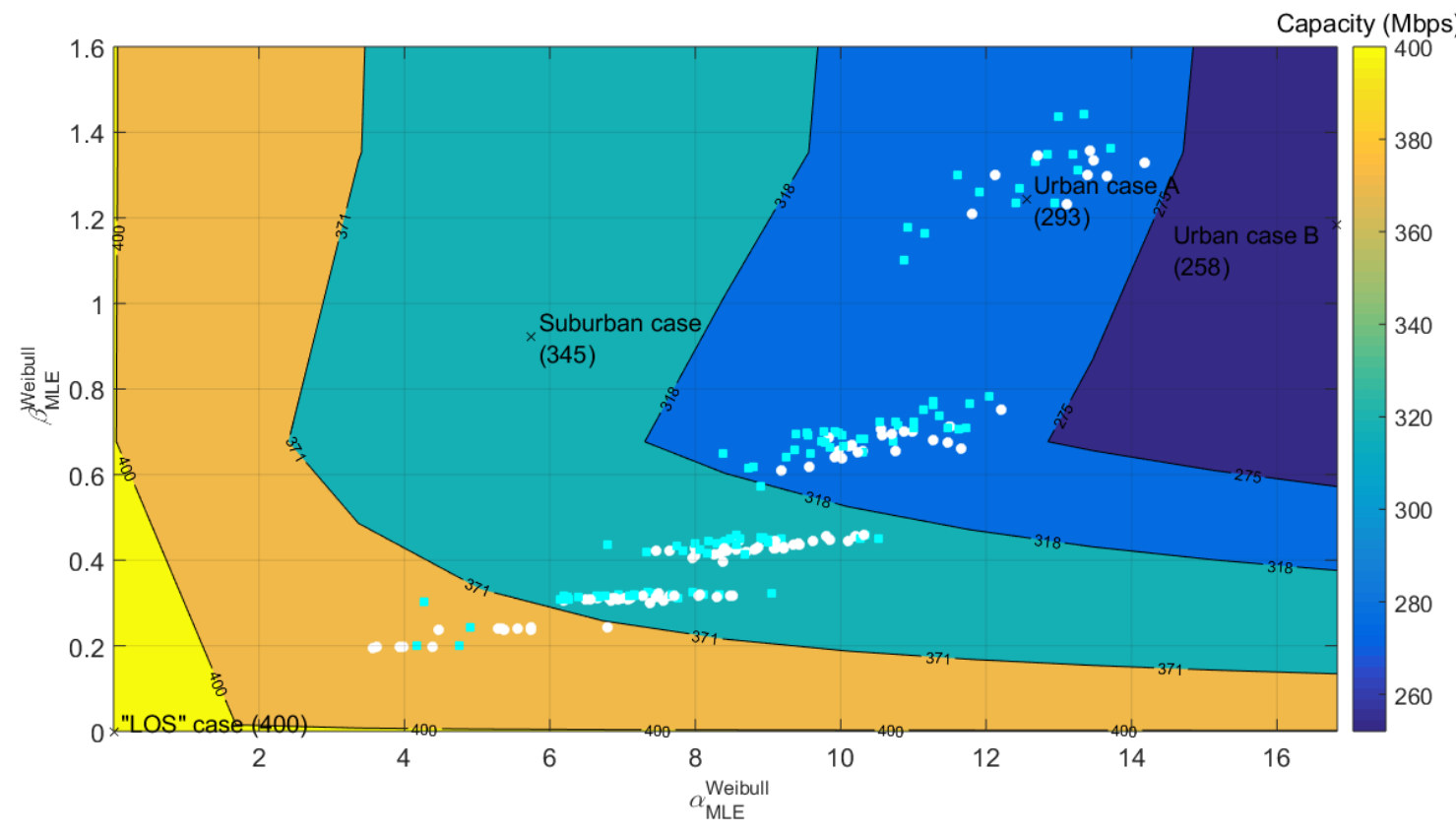

(d) 


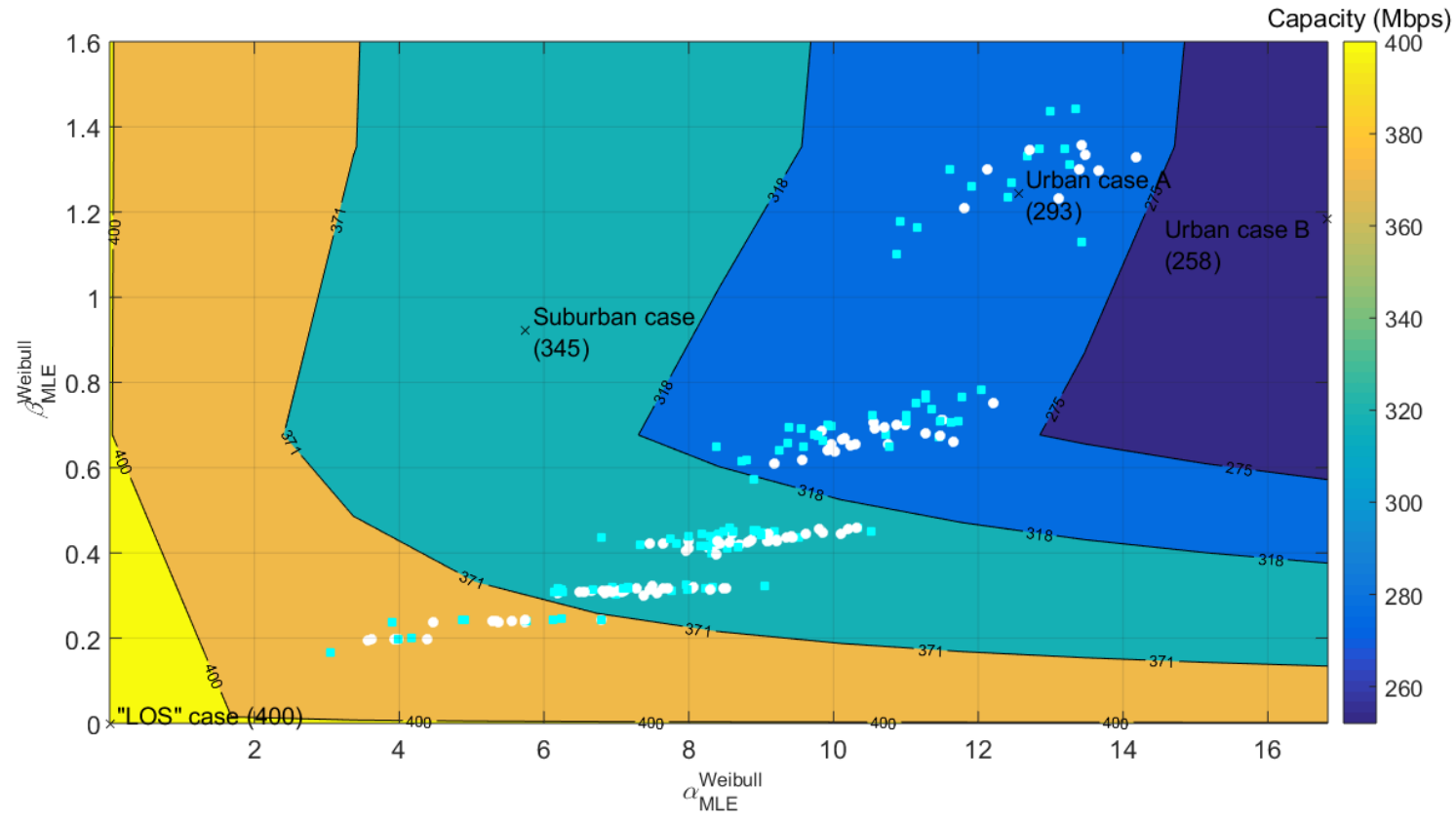

(e)

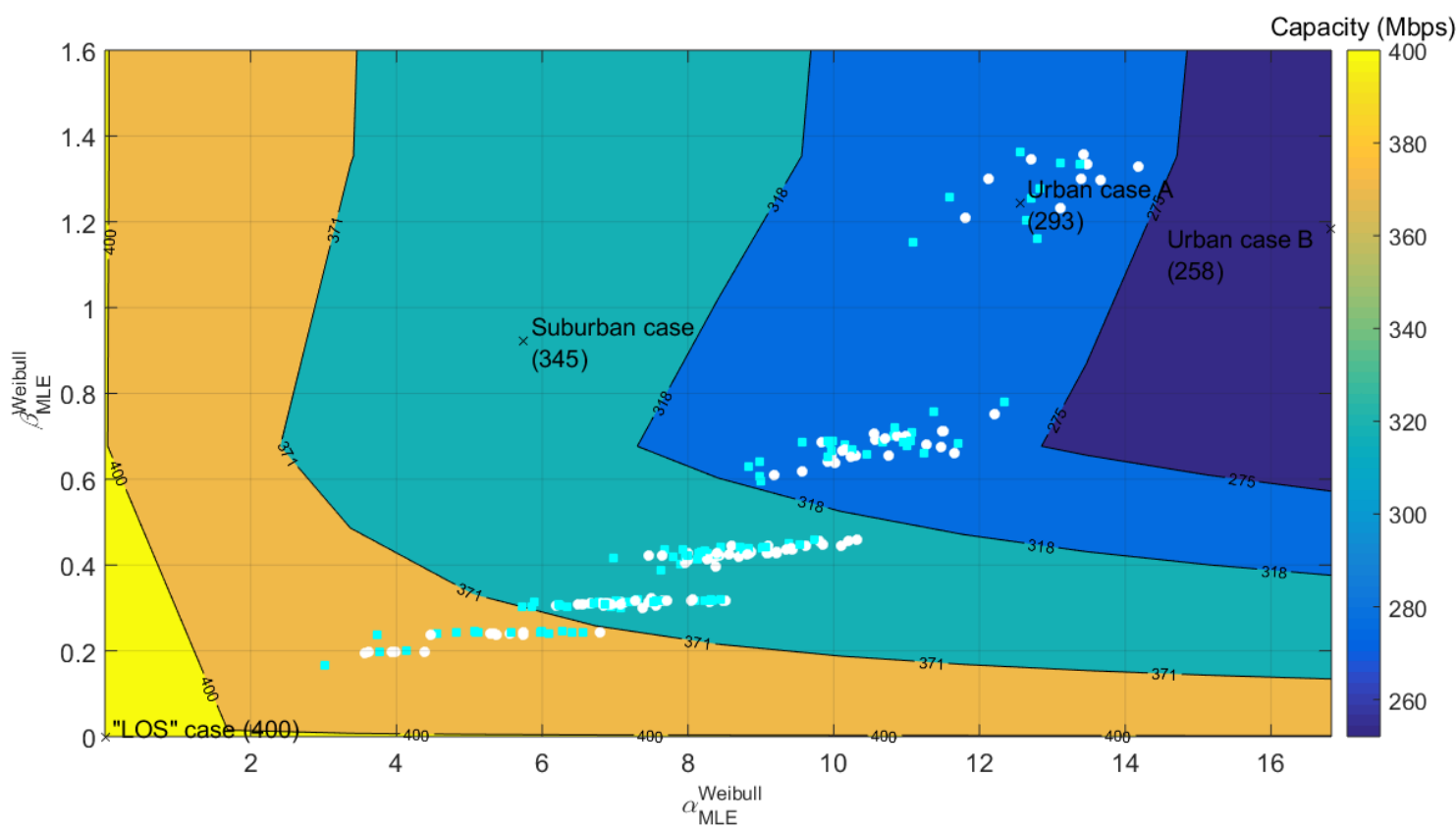

(f) 


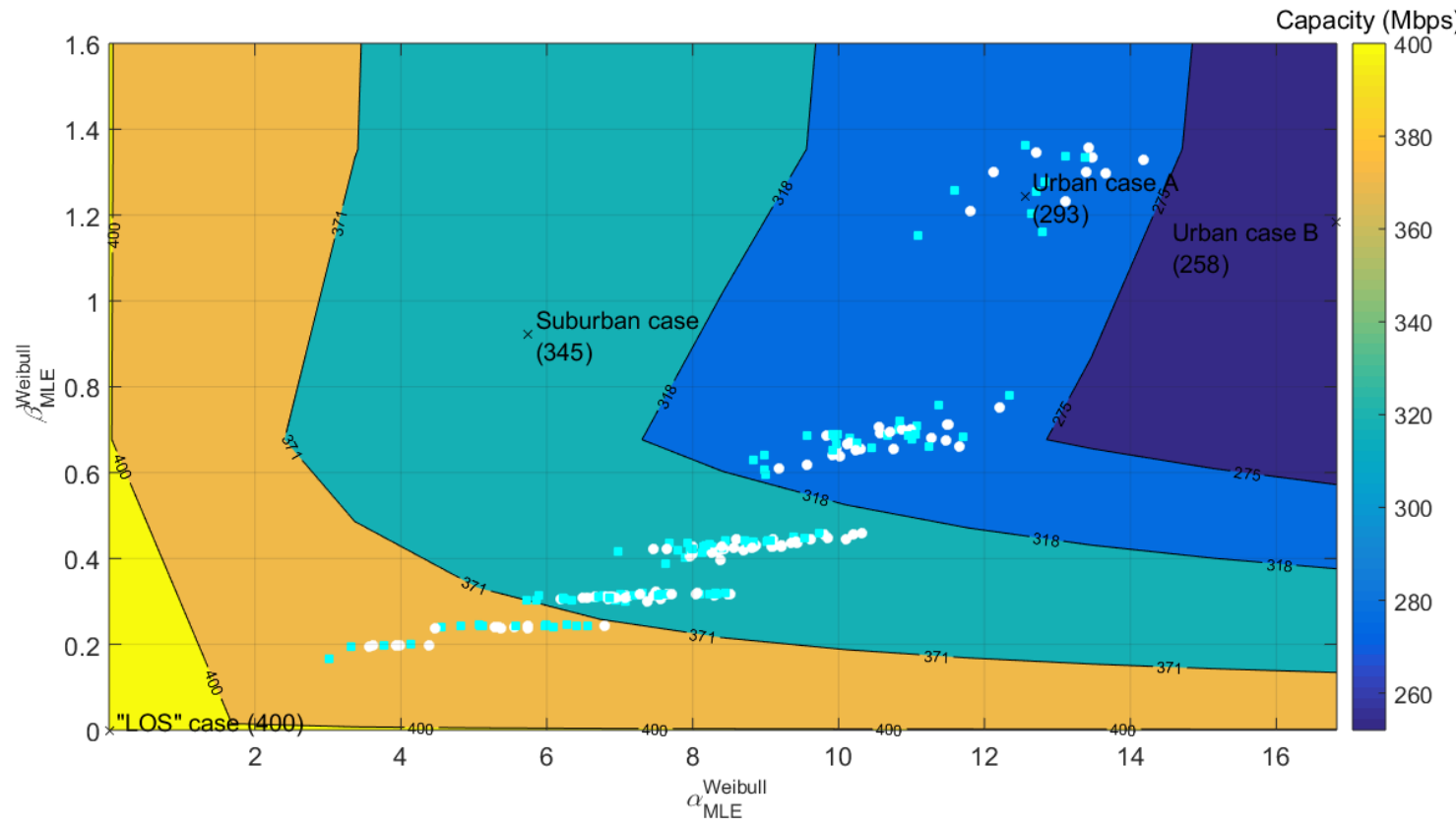

(g)

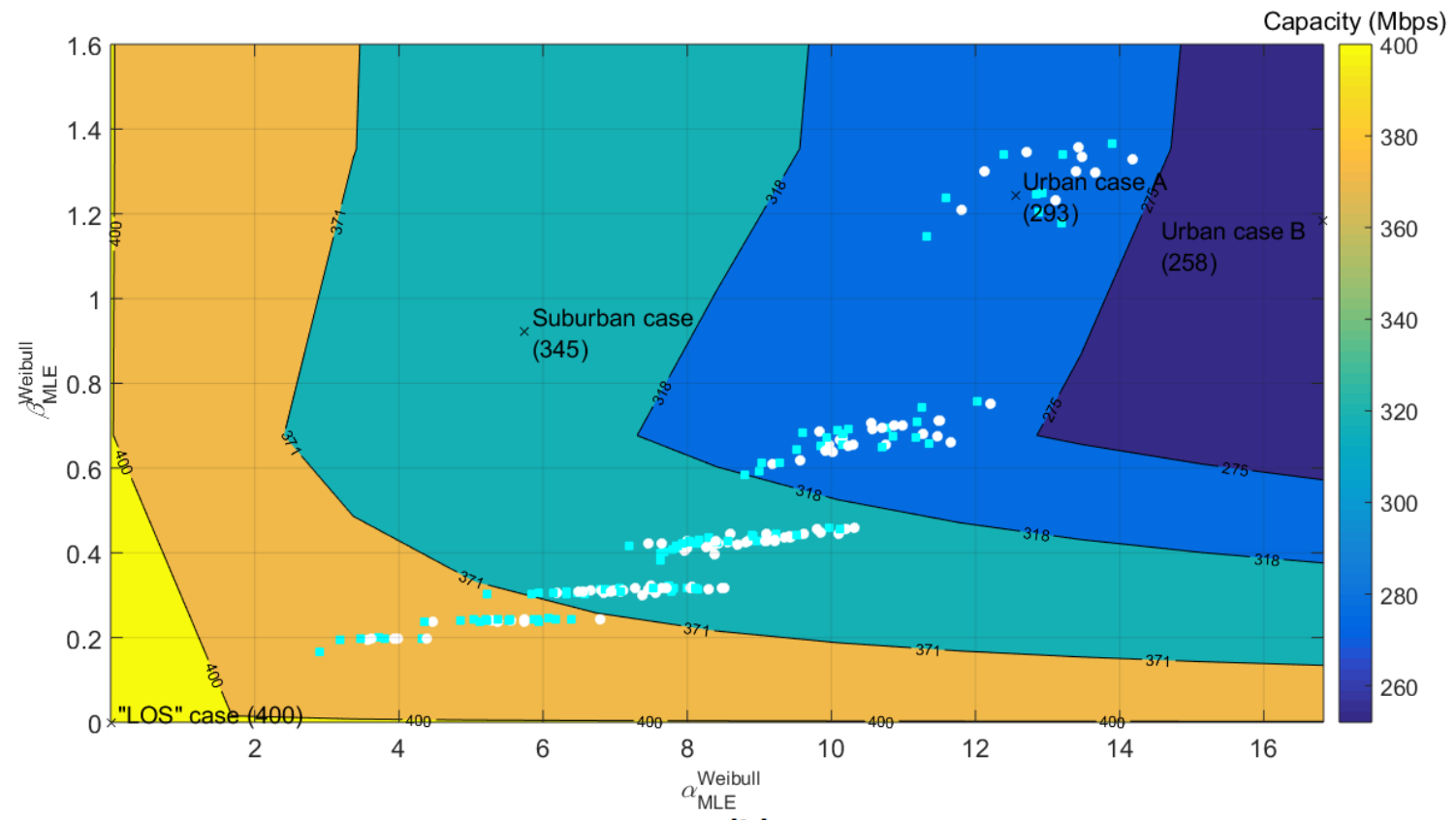

(h) 


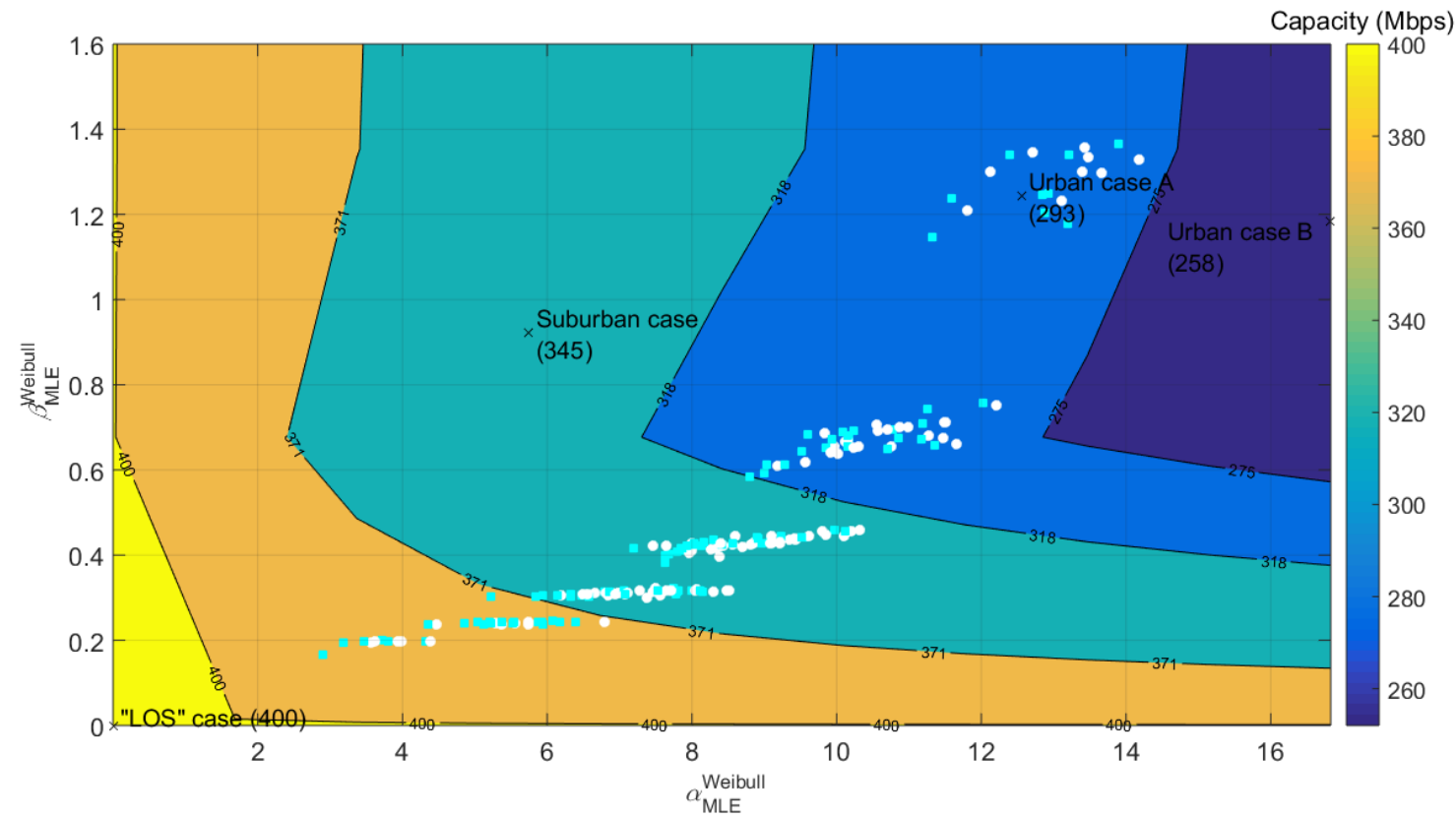

(i)

Fig. 4. iSHM footprints of the real indicative OV LV BPL urban case A when 3-30MHz frequency band, $1 \mathrm{MHz}$ frequency subchannel spacing, $\mathrm{WtG}^{1}$ coupling scheme, FCC Part 15, CUD measurement differences of maximum value $a_{\mathrm{CUD}}=6 \mathrm{~dB}$ (white circles) are assumed and various L1PMA monotonic sections (cyan squares) is applied. (a) 1 monotonic section. (b) 2 monotonic sections. (c) 3 monotonic sections. (d) 4 monotonic sections. (e) 5 monotonic sections. (f) 6 monotonic sections. (g) 7 monotonic sections. (h) 8 monotonic sections. (i) 9 monotonic sections. 


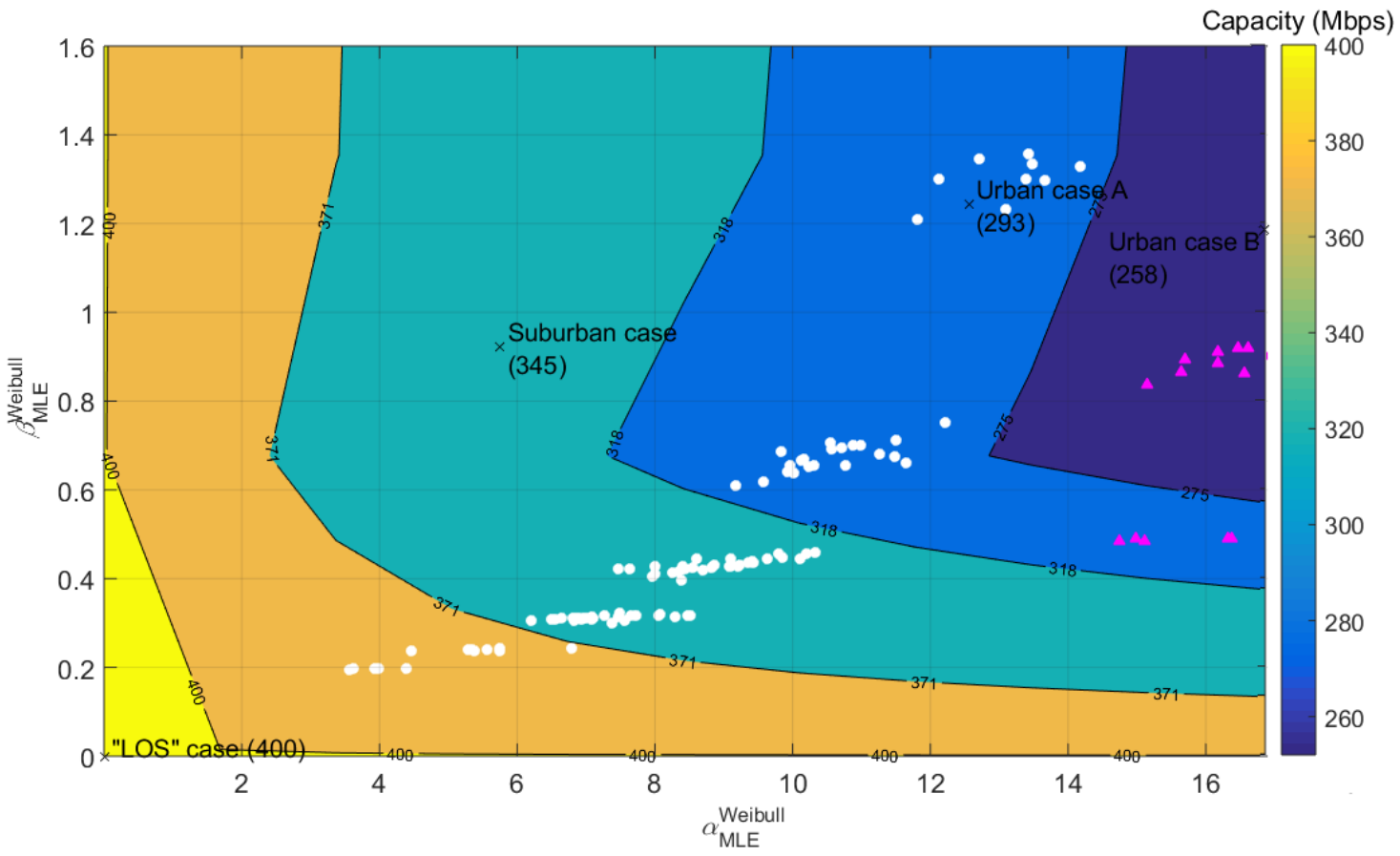

(a)

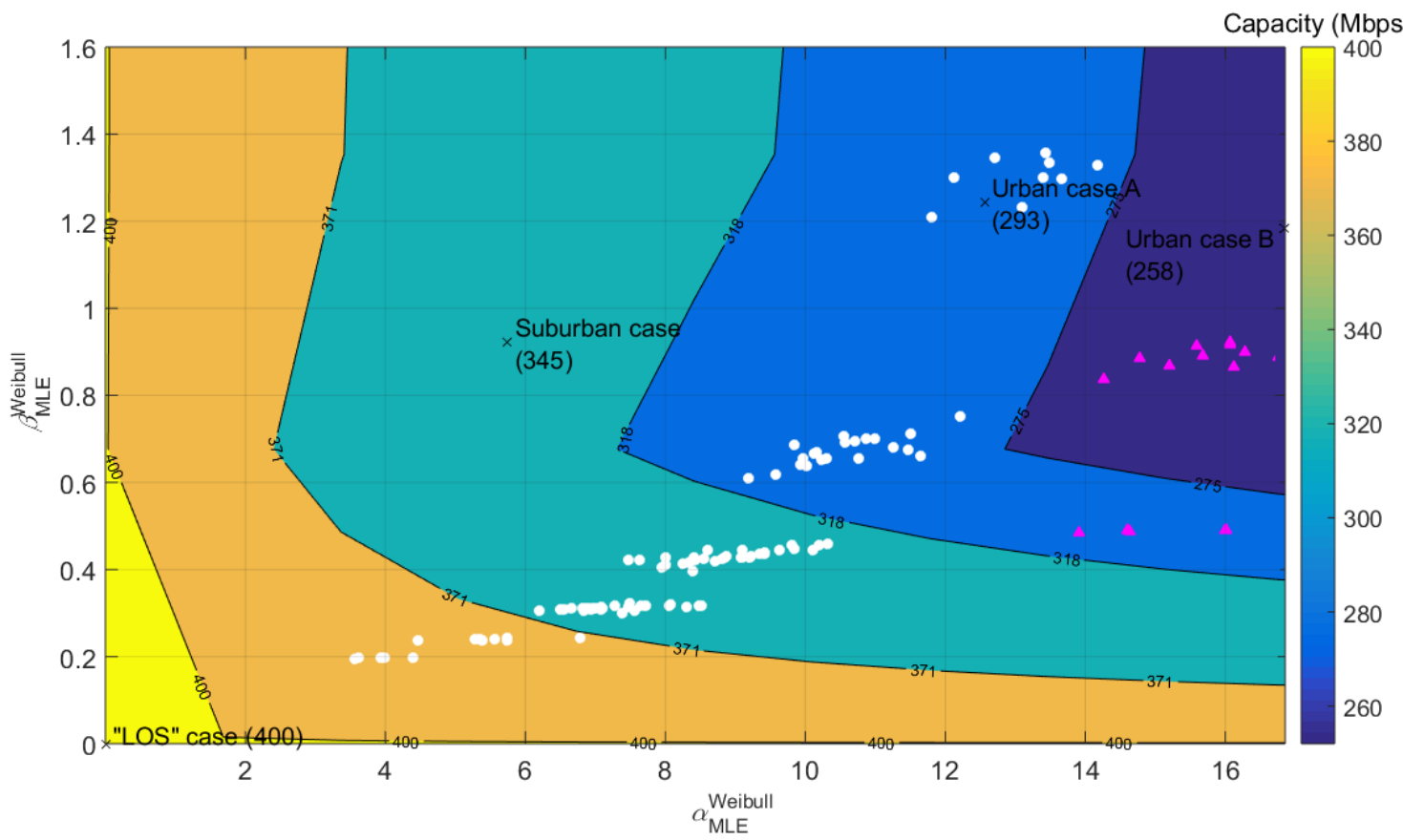

(b) 


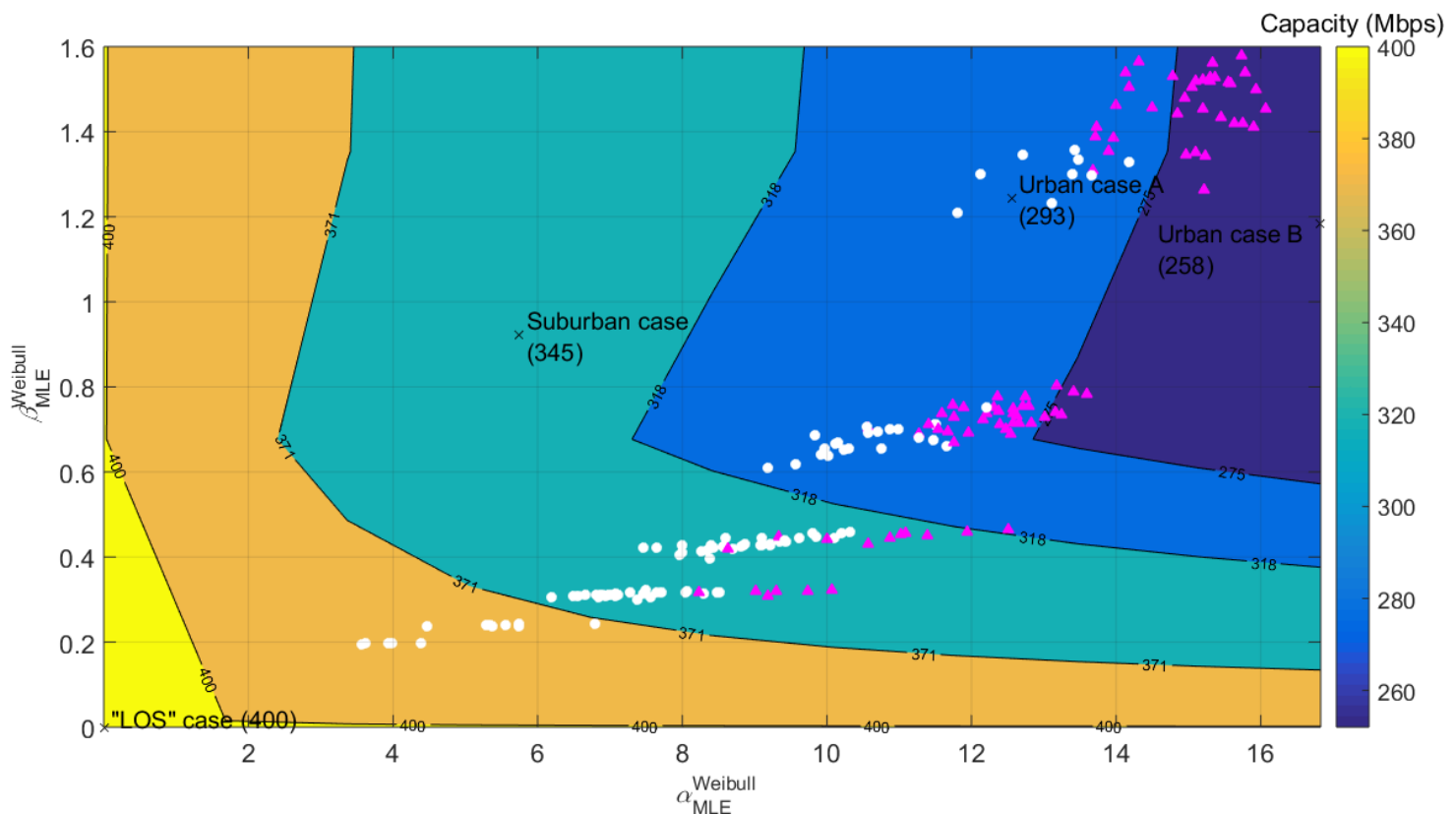

(c)

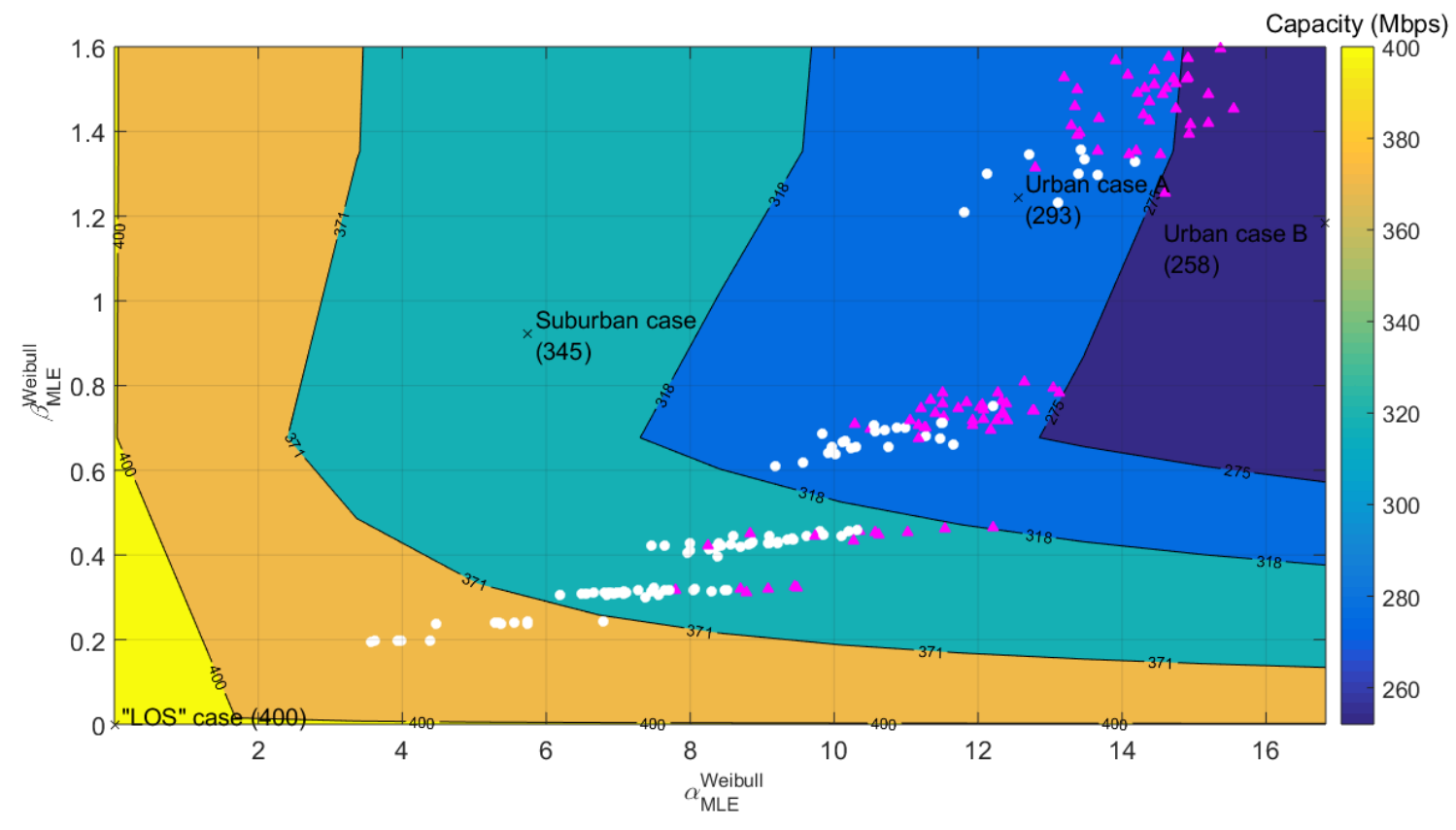

(d) 


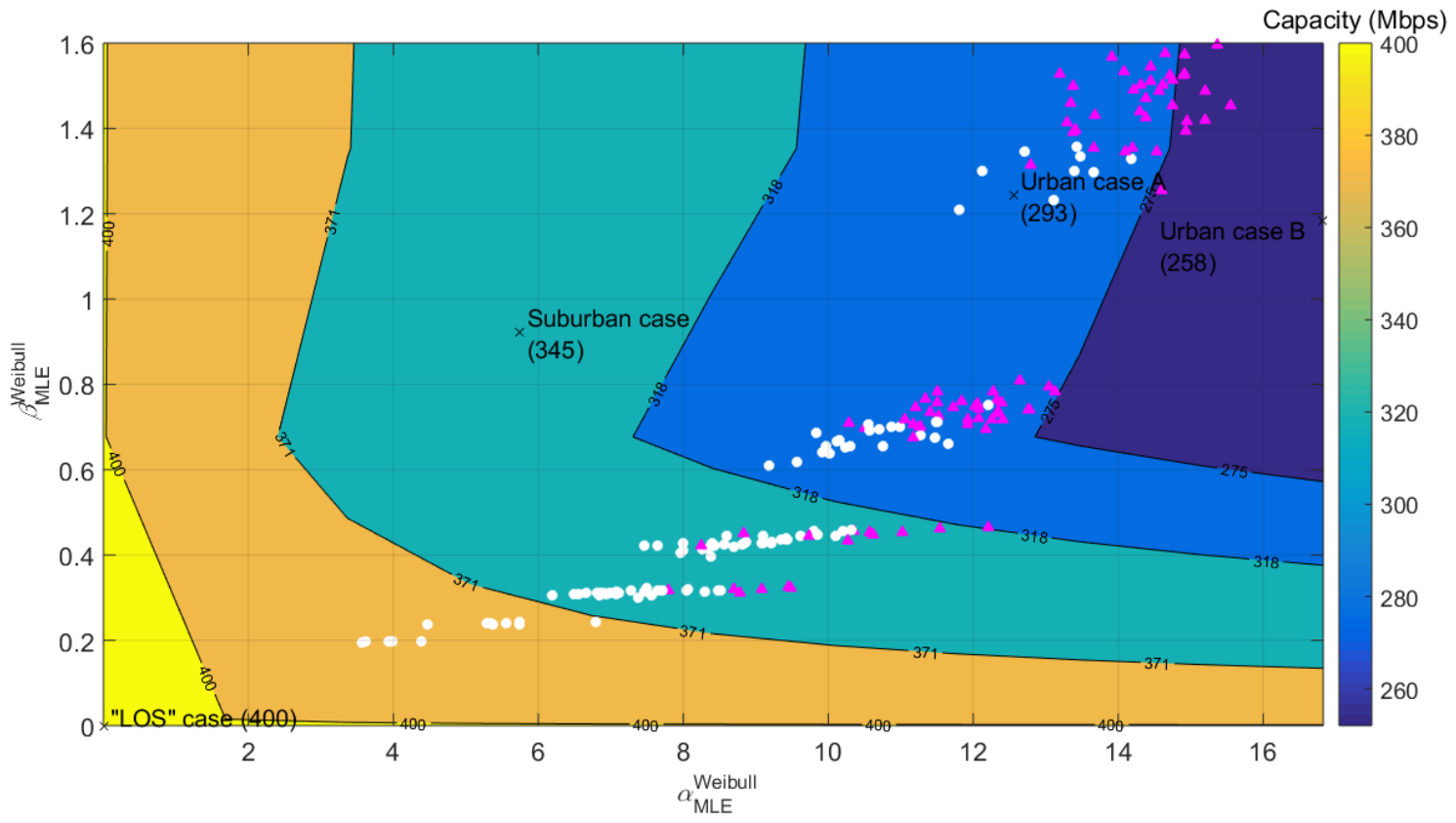

(e)

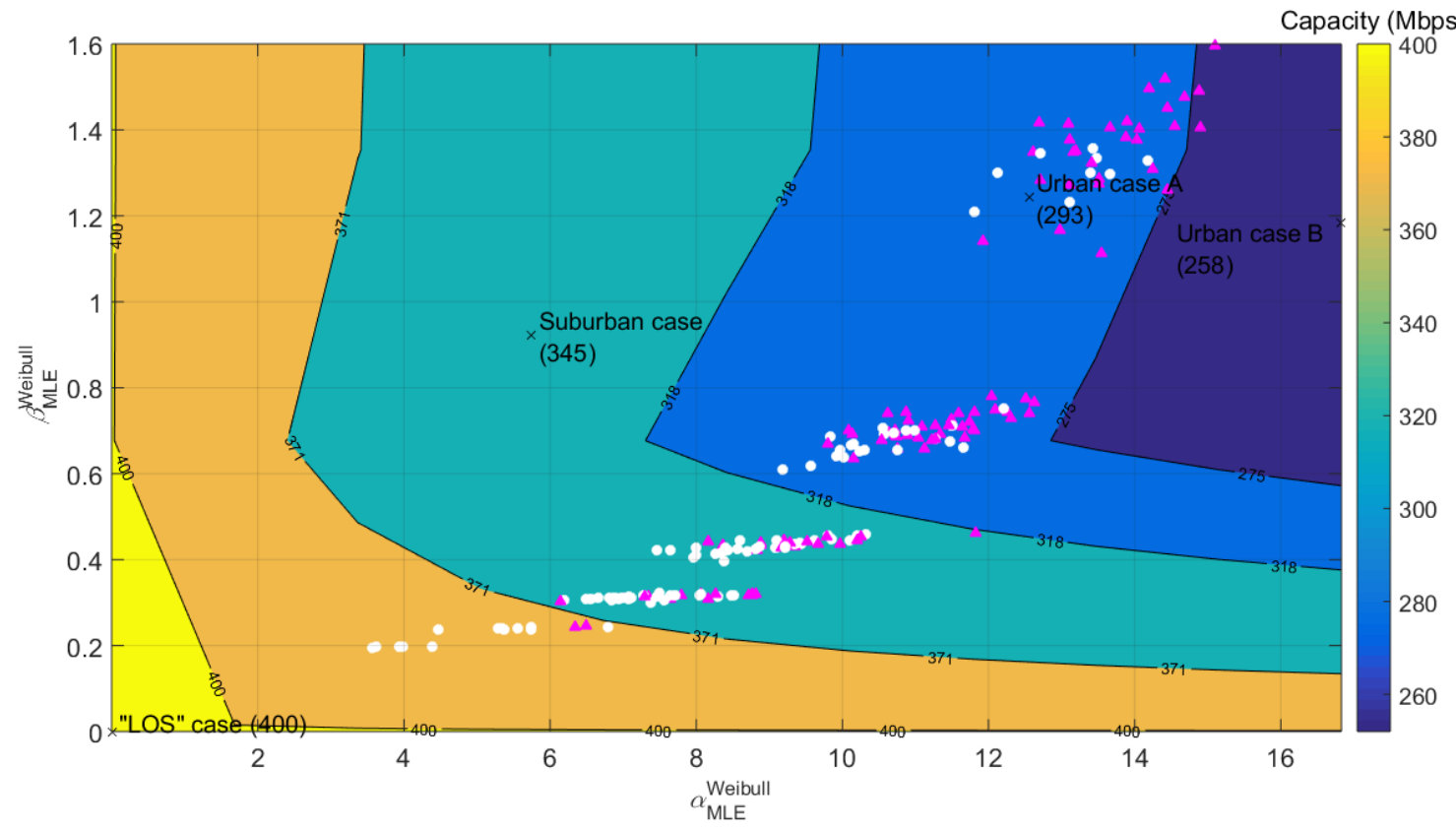

(f) 


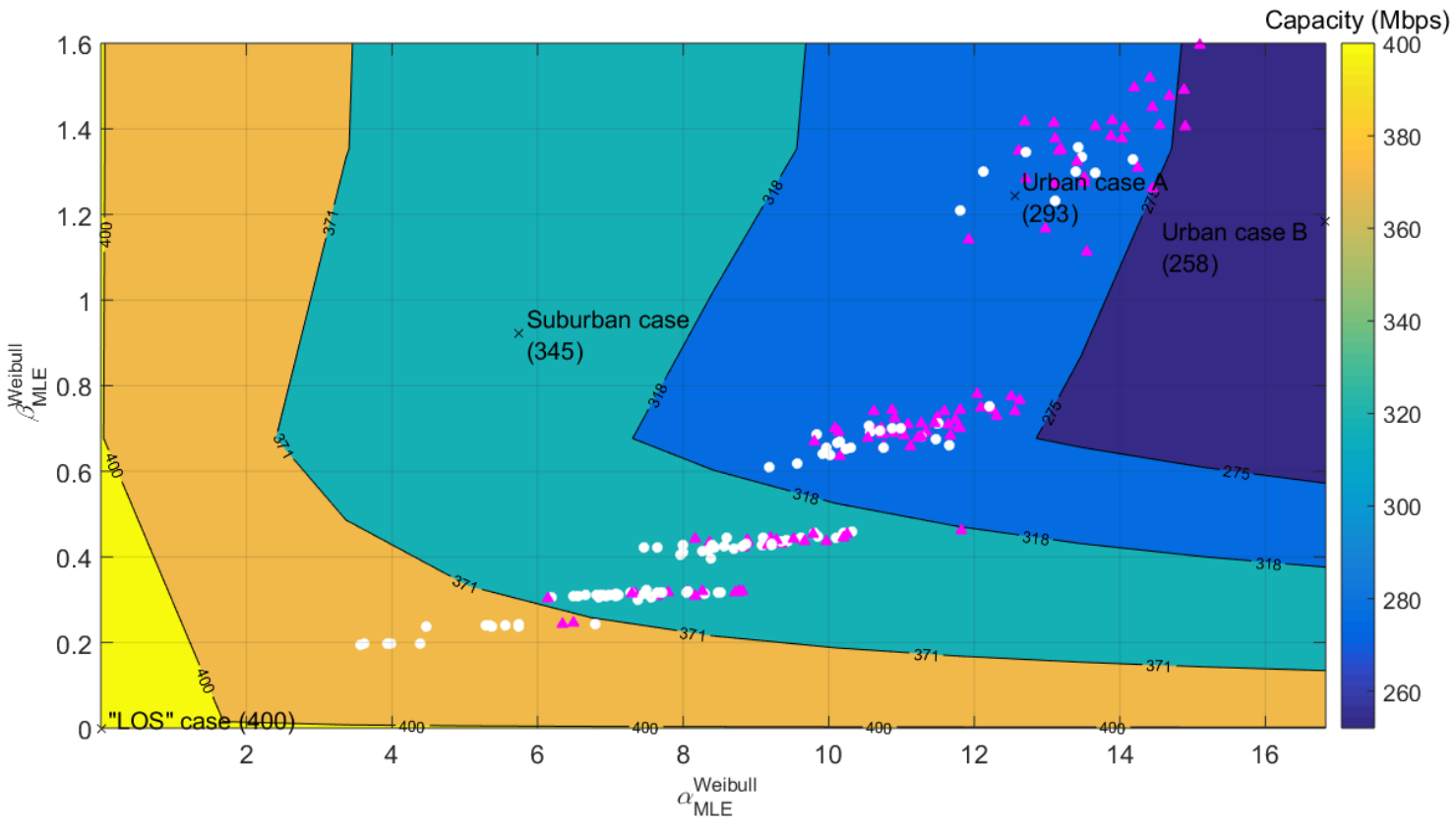

(g)

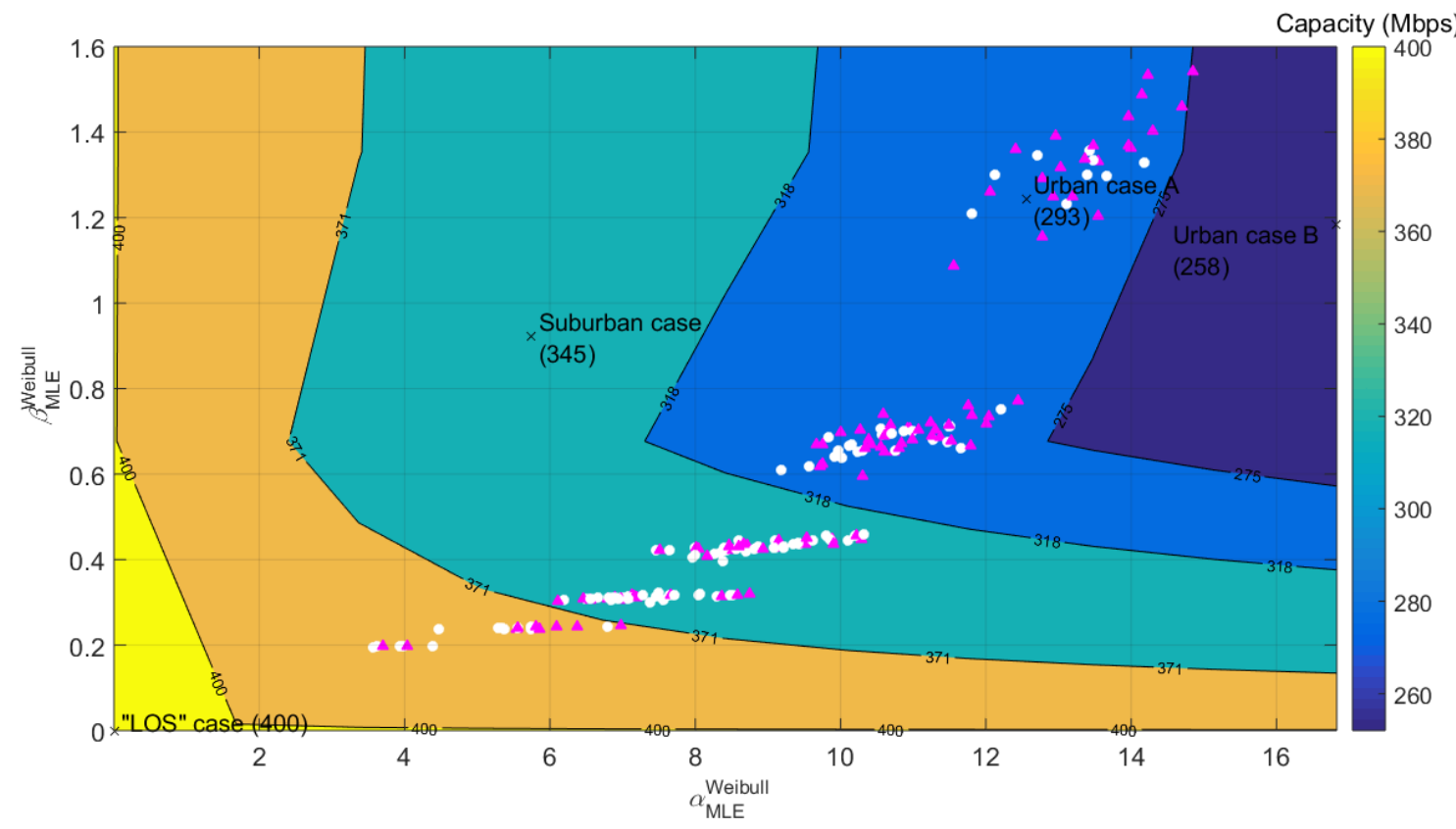

(h) 


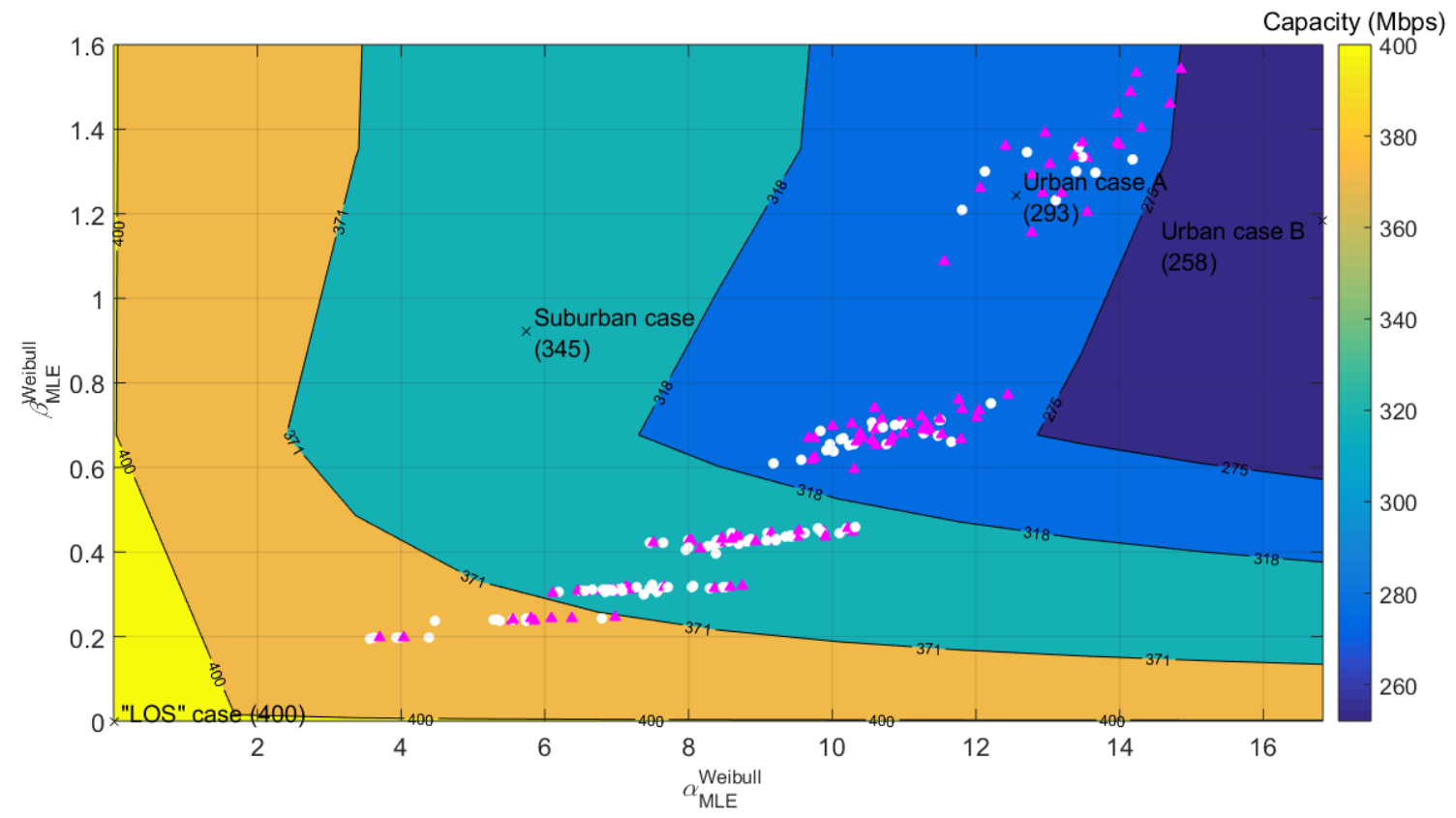

(i)

Fig. 5. Same plots with Fig. 4 but for L2WPMA of different sign changes (magenta triangles). (a) 1 sign change. (b) 2 sign changes. (c) 3 sign changes. (d) 4 sign changes. (e) 5 sign changes. (f) 6 sign changes. (g) 7 sign changes. (h) 8 sign changes. (i) 9 sign changes.

L1PMA approximations of very low monotonic sections are revealed in iSHM footprints by the significantly higher distances between the white circles and the respective cyan squares that can anyway lead to a safe mitigation of measurement differences. Same results concerning the selection of L2WPMA sign changes are observed in L2WPMA iSHM footprints. Anyway, a successful selection of L1PMA monotonic sections or L2WPMA sign changes is characterized by corresponding iSHM footprint whose cyan squares or magenta triangles are located closer to the sign of the real indicative OV LV BPL urban case A than the white circles of the measured coupling scheme transfer function data. In the rest of this paper, 1 monotonic section and 5 sign changes are assumed for the application of L1PMA and L2WPMA, respectively, by visually comparing Figs. 4(a)-(i) and 5(a)-(i), respectively.

In accordance with [36], [39], [42]-[44], the numbers of L1PMA monotonic sections and L2WPMA sign changes should be based mainly on the inherent properties of the examined theoretical coupling scheme transfer function data and secondarily on the intensity of measurement differences only for little adjustments. As the stochastic definition of the numbers of L1PMA monotonic sections and L2WPMA sign changes is applied in this paper, the aforementioned secondary dependence can be neglected. Therefore the 1 L1PMA monotonic section and 5 L2WPMA sign changes are assumed to act as constants for the following respective L1PMA and L2WPMA approximations. In the following subsection, the mitigation efficiency of L1PMA and L2WPMA against different intensities of measurement differences is qualitatively assessed through respective iSHM footprints. 


\subsection{L1PMA and L2WPMA iSHM Footprints and Different Intensities of Measurement Differences}

Higher intensities of measurement differences entail higher maximum values $a_{\text {CUD }}$ for the CUD measurement differences that are applied in this subsection. With reference to the iSHM class map of Fig. 1, the iSHM footprint due to measurement differences of the real indicative OV LV BPL urban case A is illustrated as a set of circles of various reddish colors in Fig. 6 when the maximum value $a_{\text {CUD }}$ of CUD measurement differences ranges from $0 \mathrm{~B}$ to $15 \mathrm{~dB}$. In contrast with Figs. 4(a)-(i), only one random line vector of measurement differences, say, the first one, is applied per each maximum value $a_{\text {CUD }}$ while the color of the superimposed circles becomes redder as the maximum value $a_{\text {CUD }}$ increases. Given the random line vector of measurement differences of maximum value $a_{\text {CUD }}$ and its corresponding circle of the iSHM footprint, L1PMA approximates the corresponding measured coupling scheme transfer function data with the assumed 1 monotonic section of Sec.3.3 while the respective approximated coupling scheme transfer function data are illustrated as a square of the same color with the circle color it comes from and a cyan perimeter as well as a connecting line of the same color with the circle color between the circle and the square. In Fig. 7, similar iSHM footprint with Fig. 6 is shown but for the case of L2WPMA when the 5 sign changes of Sec.3.3 are assumed and triangles with magenta perimeter are plotted instead of the L1PMA squares.

From Figs. 6 and 7, it is clear that the increasing maximum value $a_{\text {CUD }}$ of CUD measurement differences imposes the simultaneous decrease of $\hat{a}_{\mathrm{MLE}}^{\mathrm{Weibull}}$ and $\hat{\beta}_{\mathrm{MLE}}^{\text {Weibull }}$ of the measured data of the real indicative OV LV BPL urban case A. In accordance with [1], the redder circles that come from the application of higher maximum values $a_{\text {CUD }}$ are located closer to the axes origin rather than to the $\hat{a}_{\mathrm{MLE}}^{\text {Weibull }}$ and $\hat{\beta}_{\mathrm{MLE}}^{\mathrm{Weibull}}$ of the theoretical data of the real indicative OV LV BPL urban case A. Hence, the iSHM footprint due to the increasing measurement differences starts from the neighborhood of the theoretical values of $\hat{a}_{\mathrm{MLE}}^{\text {Weibull }}$ and $\hat{\beta}_{\mathrm{MLE}}^{\text {Weibull }}$ of the real indicative OV LV BPL urban case A and tends to the axes origin in a down-left diagonal direction. The goal of the application of piecewise monotonic data approximations is to reverse the previous diagonal direction of red circles and to bring the approximated respective red L1PMA squares and L2WPMA triangles: (i) back to the OV LV BPL urban case A topology class as primary objective; and (ii) as close as possible to the theoretical values of $\hat{a}_{\mathrm{MLE}}^{\mathrm{Weibull}}$ and $\hat{\beta}_{\mathrm{MLE}}^{\mathrm{Weibull}}$ as secondary but more accurate objective.

In order to qualitatively assess the performance of L1PMA and L2WPMA against the increasing measurement differences, the location and the distance of L1PMA squares and L2WPMA triangles that come from the respective circles of the measured data are tracked. In the vast majority of the cases, squares and triangles are located closer to the theoretical values of $\hat{a}_{\mathrm{MLE}}^{\text {Weibull }}$ and $\hat{\beta}_{\mathrm{MLE}}^{\text {Weibull }}$ of the real indicative OV LV BPL urban case A compared against the respective circles of the measured data. More analytically, in the examined cases where high measurement differences are applied, the L1PMA mitigation of measurement differences is important since from the six circles that are located at $\mathrm{OV}$ LV BPL rural and "LOS" topology classes, no squares remain inside the aforementioned classes. Similarly, L2WPMA countermeasures against measurement differences achieve to mitigate five out of the five circles that are located at OV LV BPL rural and 


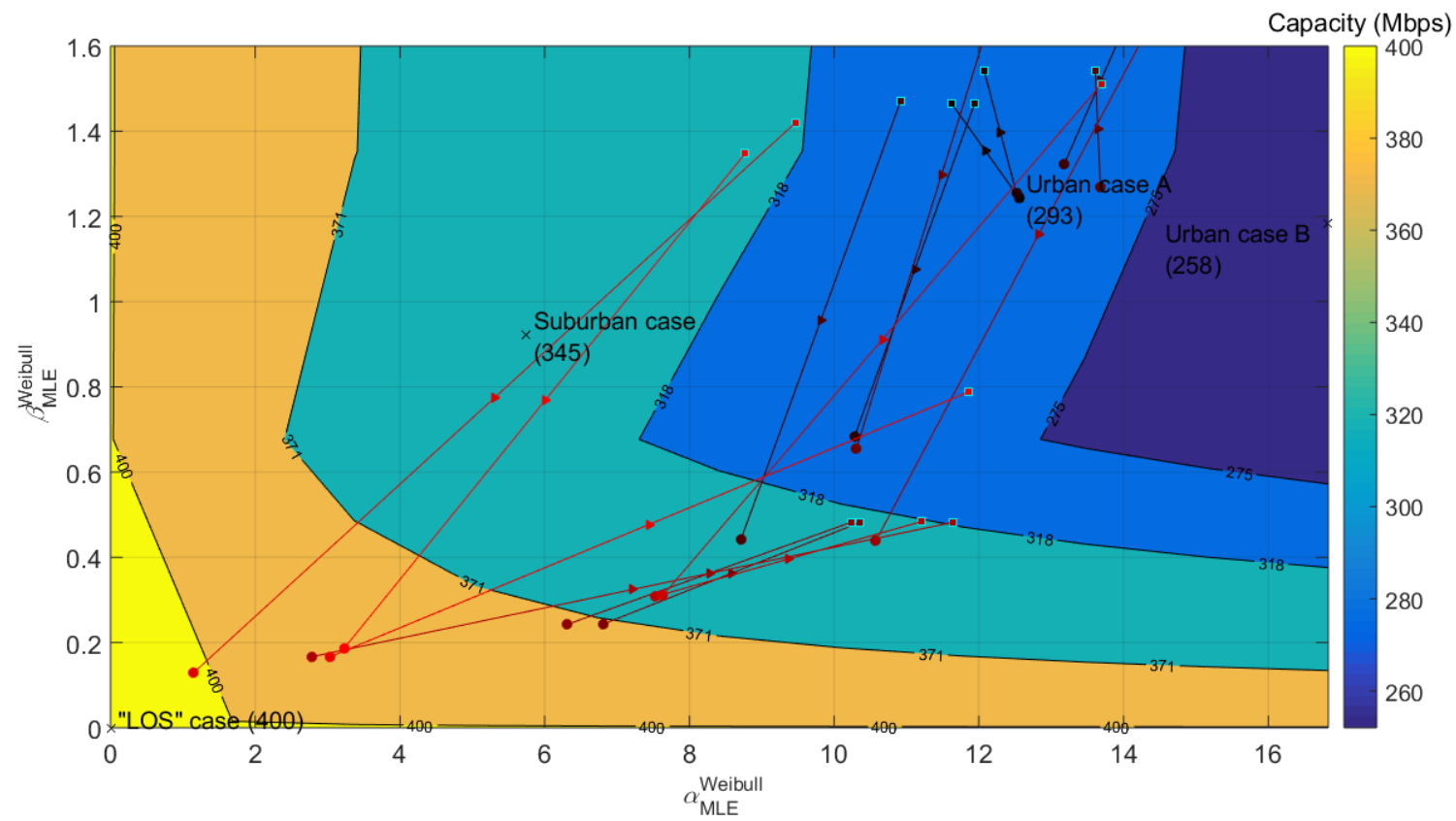

Fig. 6. iSHM footprints of the real indicative OV LV BPL topologies when 3-30MHz frequency band, $\mathrm{WtG}^{1}$ coupling scheme, FCC Part 15, 1 monotonic section of L1PMA and CUD measurement differences of maximum value $a_{\mathrm{CUD}}$ that ranges from $0 \mathrm{~dB}$ (black spot) to $15 \mathrm{~dB}$ (red spot) are assumed.

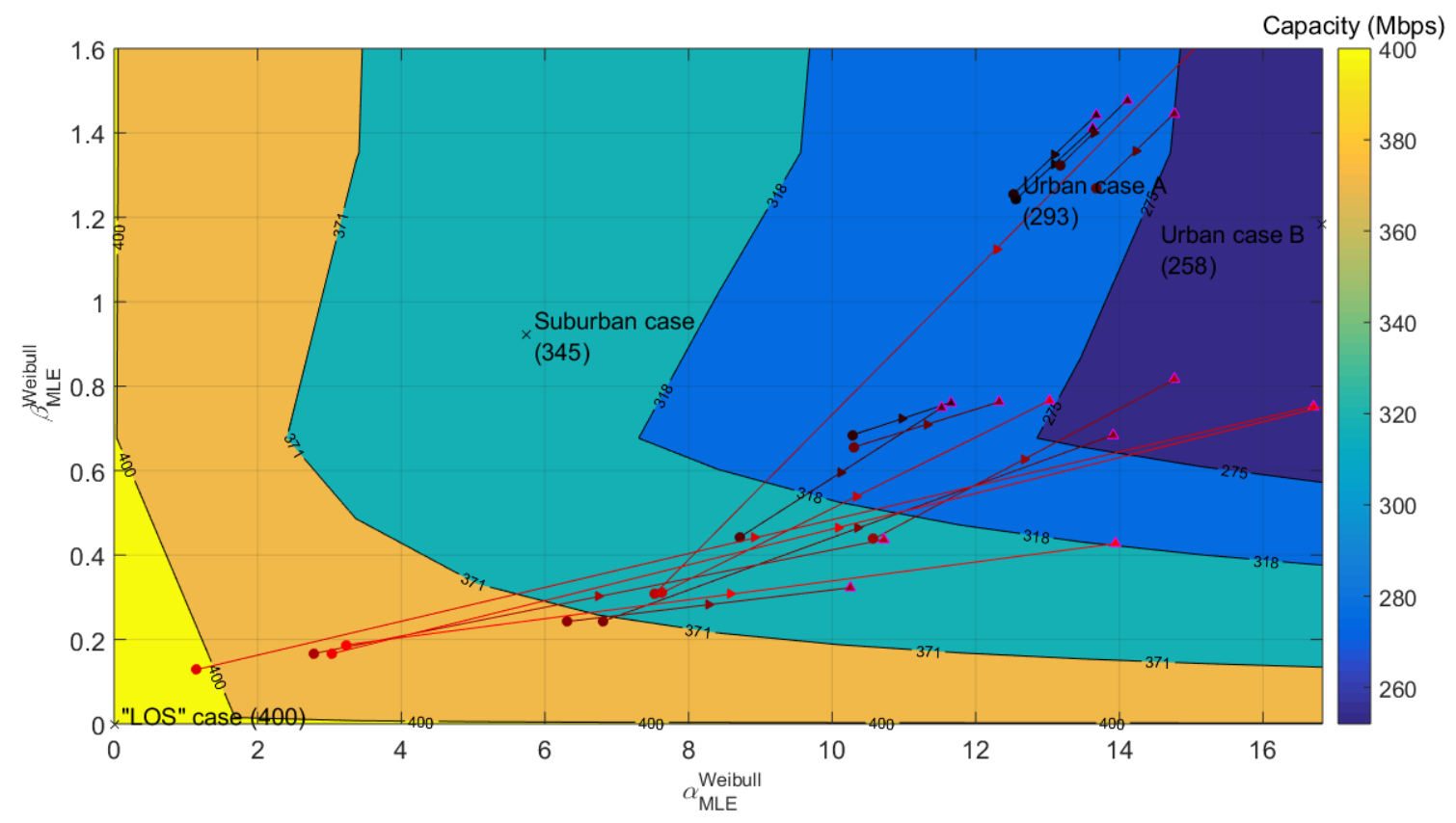

Fig. 7. Same plots but for 5 sign changes of L2WPMA.

"LOS" topology classes. In addition, from the 16 available circles, 6 of them are located at the OV LV BPL urban case A topology class when 8 squares and 9 triangles are present in the visible part of the previous class after the application of L1PMA and L2WPMA, respectively. Anyway, the connecting lines with their triangle signs at their middle reveal the performance of L1PMA and L2WPMA against the measurement differences while the mitigation efficiency becomes more significant when measurement 
differences are higher. Anyway, the promising results regarding the mitigation of higher measurement differences by L1PMA and L2WPMA was expected after the determination of respective monotonic sections and sign changes in Sec.3.3.

In this subsection, the qualitative assessment of piecewise monotonic data approximations via iSHM footprints has revealed their strong potential against measurement differences of increasing intensity. The obvious evolution of this piece of research is the proposal of a quantitative assessment of the mitigation impact of piecewise monotonic data approximations based on iSHM footprints in [53] so that a more confident selection of L1PMA monotonic sections and L2WPMA sign changes can be achieved.

\section{Conclusions}

To ensure the reliability of data that feed the business analytics and the tools of the SG, the mitigation performance of L1PMA and L2WPMA against measurement differences has been qualitatively assessed via iSHM footprints for OV LV BPL topologies. Indeed, L1PMA and L2WPMA iSHM footprints present significant improvement concerning their extent and their distance from Weibull CASD MLEs of the real indicative OV LV BPL urban case A when they are compared against iSHM footprints due to measurement differences. Also, it has been verified that the fine selection of L1PMA monotonic sections and L2WPMA sign changes plays critical role towards a successful mitigation of measurement differences. In addition, the proposed countermeasures of applying L1PMA and L2WPMA have been proven to be a valuable tool against high measurement differences since the mitigation efficiency in these cases is important. Finally, the qualitative assessment of piecewise monotonic data approximations has been validated via iSHM footprints but the continuation of the research in [53] focuses on the proposal of quantitative metrics for iSHM footprints that allows the more precise selection of L1PMA monotonic sections and L2WPMA sign changes on the basis of the available iSHM footprints.

\section{CONFLICTS OF INTEREST}

The author declares that there is no conflict of interests regarding the publication of this paper.

\section{References}

[1] A. G. Lazaropoulos, "Business Analytics and IT in Smart Grid - Part 1: The Impact of Measurement Differences on the iSHM Class Map Footprints of Overhead Low-Voltage Broadband over Power Lines Topologies," Trends in Renewable Energy, vol. 6, no. 2, pp. 146-176, May 2020.

[2] A. G. Lazaropoulos, "Factors Influencing Broadband Transmission Characteristics of Underground Low-Voltage Distribution Networks," IET Commun., vol. 6, no. 17, pp. 2886-2893, Nov. 2012. 
[3] F. Aalamifar and L. Lampe, "Optimized WiMAX profile configuration for smart grid communications," IEEE Transactions on Smart Grid, vol. 8, no. 6, pp. 27232732, 2017.

[4] A. G. Lazaropoulos, "Deployment Concepts for Overhead High Voltage Broadband over Power Lines Connections with Two-Hop Repeater System: Capacity Countermeasures against Aggravated Topologies and High Noise Environments," Progress in Electromagnetics Research B, vol. 44, pp. 283-307, 2012. [Online]. Available: http://www.jpier.org/PIERB/pierb44/13.12081104.pdf

[5] A. G. Lazaropoulos, "Towards Broadband over Power Lines Systems Integration: Transmission Characteristics of Underground Low-Voltage Distribution Power Lines," Progress in Electromagnetics Research B, vol. 39, pp. 89-114, 2012. [Online]. Available: http://www.jpier.org/PIERB/pierb39/05.12012409.pdf

[6] A. G. Lazaropoulos, "Broadband Performance Metrics and Regression Approximations of the New Coupling Schemes for Distribution Broadband over Power Lines (BPL) Networks," Trends in Renewable Energy, vol. 4, no. 1, pp. 43-73, Jan. $2018 . \quad$ [Online]. Available: http://futureenergysp.com/index.php/tre/article/view/59/pdf

[7] A. G. Lazaropoulos, "Wireless Sensor Network Design for Transmission Line Monitoring, Metering and Controlling Introducing Broadband over PowerLinesenhanced Network Model (BPLeNM)," ISRN Power Engineering, vol. 2014, Article ID 894628, 22 pages, 2014. doi:10.1155/2014/894628. [Online]. Available:

http://www.hindawi.com/journals/isrn.power.engineering/2014/894628/

[8] A. G. Lazaropoulos, "Improvement of Power Systems Stability by Applying Topology Identification Methodology (TIM) and Fault and Instability Identification Methodology (FIIM)-Study of the Overhead Medium-Voltage Broadband over Power Lines (OV MV BPL) Networks Case," Trends in Renewable Energy, vol. 3, no. 2, pp. 102-128, Apr. 2017. [Online]. Available: http://futureenergysp.com/index.php/tre/article/view/34

[9] A. G. Lazaropoulos, "Main Line Fault Localization Methodology in Smart GridPart 1: Extended TM2 Method for the Overhead Medium-Voltage Broadband over Power Lines Networks Case," Trends in Renewable Energy, vol. 3, no. 3, pp. 2-25, Dec. $2017 . \quad$ [Online]. Available: http://futureenergysp.com/index.php/tre/article/view/36

[10] A. G. Lazaropoulos, "Main Line Fault Localization Methodology in Smart GridPart 2: Extended TM2 Method, Measurement Differences and L1 Piecewise Monotonic Data Approximation for the Overhead Medium-Voltage Broadband over Power Lines Networks Case," Trends in Renewable Energy, vol. 3, no. 3, pp. 26-61, Dec. 2017. [Online]. Available: http://futureenergysp.com/index.php/tre/article/view/37

[11] A. G. Lazaropoulos, "Main Line Fault Localization Methodology in Smart GridPart 3: Main Line Fault Localization Methodology (MLFLM)," Trends in Renewable Energy, vol. 3, no. 3, pp. 62-81, Dec. 2017. [Online]. Available: http://futureenergysp.com/index.php/tre/article/view/38

[12] A. G. Lazaropoulos, "Main Line Fault Localization Methodology (MLFLM) in Smart Grid-The Underground Medium- and Low-Voltage Broadband over Power Lines Networks Case," Trends in Renewable Energy, vol. 4, no. 1, pp. 15-42, 
Dec. 2017. [Online]. Available: http://futureenergysp.com/index.php/tre/article/view/45

[13] A. G. Lazaropoulos, "Smart Energy and Spectral Efficiency (SE) of Distribution Broadband over Power Lines (BPL) Networks - Part 1: The Impact of Measurement Differences on SE Metrics," Trends in Renewable Energy, vol. 4, no. 2, pp. 125-184, Aug. 2018. [Online]. Available: http://futureenergysp.com/index.php/tre/article/view/76/pdf

[14] A. G. Lazaropoulos, "Smart Energy and Spectral Efficiency (SE) of Distribution Broadband over Power Lines (BPL) Networks - Part 2: L1PMA, L2WPMA and L2CXCV for SE against Measurement Differences in Overhead Medium-Voltage BPL Networks," Trends in Renewable Energy, vol. 4, no. 2, pp. 185-212, Aug. 2018. [Online]. Available: http://futureenergysp.com/index.php/tre/article/view/77/pdf

[15] A. G. Lazaropoulos, "Underground Distribution BPL Connections with $(\mathrm{N}+1)$ hop Repeater Systems: A Novel Capacity Mitigation Technique," Elsevier Computers and Electrical Engineering, vol. 40, pp. 1813-1826, 2014.

[16] A. G. Lazaropoulos, "Review and Progress towards the Capacity Boost of Overhead and Underground Medium-Voltage and Low-Voltage Broadband over Power Lines Networks: Cooperative Communications through Two- and ThreeHop Repeater Systems," ISRN Electronics, vol. 2013, Article ID 472190, pp. 119, $2013 . \quad$ [Online]. Available: http://www.hindawi.com/isrn/electronics/aip/472190/

[17] A. G. Lazaropoulos, "Broadband over Power Lines (BPL) Systems Convergence: Multiple-Input Multiple-Output (MIMO) Communications Analysis of Overhead and Underground Low-Voltage and Medium-Voltage BPL Networks (Invited Paper)," ISRN Power Engineering, vol. 2013, Article ID 517940, pp. 1-30, 2013. [Online].

Available: http://www.hindawi.com/isrn/power.engineering/2013/517940/

[18] A. Nazem and M. R Arshad, "An Approach in Full Duplex Digital Multipoint Systems Using Large Signal Power Line Communication," Bentham Recent Patents on Electrical \& Electronic Engineering, vol. 6, no. 2, pp. 138-146, 2013.

[19] A. G. Lazaropoulos and P. G. Cottis, "Transmission characteristics of overhead medium voltage power line communication channels," IEEE Trans. Power Del., vol. 24, no. 3, pp. 1164-1173, Jul. 2009.

[20] A. G. Lazaropoulos and P. G. Cottis, "Capacity of overhead medium voltage power line communication channels," IEEE Trans. Power Del., vol. 25, no. 2, pp. 723-733, Apr. 2010.

[21] A. G. Lazaropoulos and P. G. Cottis, "Broadband transmission via underground medium-voltage power lines-Part I: transmission characteristics," IEEE Trans. Power Del., vol. 25, no. 4, pp. 2414-2424, Oct. 2010.

[22] A. G. Lazaropoulos and P. G. Cottis, "Broadband transmission via underground medium-voltage power lines-Part II: capacity," IEEE Trans. Power Del., vol. 25, no. 4 , pp. 2425-2434, Oct. 2010.

[23] A. G. Lazaropoulos, "Broadband transmission and statistical performance properties of overhead high-voltage transmission networks," Hindawi Journal of Computer Networks and Commun., 2012, article ID 875632, 2012. [Online]. Available: http://www.hindawi.com/journals/jcnc/aip/875632/ 
[24] A. G. Lazaropoulos, "Statistical Channel Modeling of Overhead Low Voltage Broadband over Power Lines (OV LV BPL) Networks - Part 1: The Theory of Class Map Footprints of Real OV LV BPL Topologies, Branch Line Faults and Hook-Style Energy Thefts," Trends in Renewable Energy, vol. 6, no. 1, pp. 61-87, Mar. 2020. [Online]. Available: http://futureenergysp.com/index.php/tre/article/download/112/pdf

[25] E. Biglieri, "Coding and modulation for a horrible channel," IEEE Commun. Mag., vol. 41, no. 5, pp. 92-98, May 2003.

[26] M. Gebhardt, F. Weinmann, and K. Dostert, "Physical and regulatory constraints for communication over the power supply grid," IEEE Commun. Mag., vol. 41, no. 5, pp. 84-90, May 2003.

[27] M. Götz, M. Rapp, and K. Dostert, "Power line channel characteristics and their effect on communication system design," IEEE Commun. Mag., vol. 42, no. 4, pp. 78-86, Apr. 2004.

[28] A. G. Lazaropoulos, "Towards Modal Integration of Overhead and Underground Low-Voltage and Medium-Voltage Power Line Communication Channels in the Smart Grid Landscape: Model Expansion, Broadband Signal Transmission Characteristics, and Statistical Performance Metrics (Invited Paper)," ISRN Signal Processing, vol. 2012, Article ID 121628, pp. 1-17, 2012. [Online]. Available: http://www.hindawi.com/isrn/sp/2012/121628/

[29] A. G. Lazaropoulos, "Statistical Broadband over Power Lines Channel Modeling - Part 1: The Theory of the Statistical Hybrid Model," Progress in Electromagnetics Research C, vol. 92, pp. 1-16, 2019. [Online]. Available: http://www.jpier.org/PIERC/pierc92/01.19012902.pdf

[30] A. G. Lazaropoulos, "Statistical Broadband over Power Lines (BPL) Channel Modeling - Part 2: The Numerical Results of the Statistical Hybrid Model," Progress in Electromagnetics Research C, vol. 92, pp. 17-30, 2019. [Online]. Available: http://www.jpier.org/PIERC/pierc92/02.19012903.pdf

[31] A. G. Lazaropoulos, "Enhancing the Statistical Hybrid Model Performance in Overhead and Underground Medium Voltage Broadband over Power Lines Channels by Adopting Empirical Channel Attenuation Statistical Distribution," Trends in Renewable Energy, vol. 5, no. 2, pp. 181-217, 2019. [Online]. Available: http://futureenergysp.com/index.php/tre/article/view/96/pdf

[32] F. Pancaldi, F. Gianaroli, and G. M. Vitetta, "Equalization of Narrowband Indoor Powerline Channels for High Data Rate OFDM Communications," IEEE Trans. on Smart Grid, vol. 9, no. 1, pp. 78-87, Jan. 2018.

[33] A. G. Lazaropoulos, "Virtual Indicative Broadband over Power Lines Topologies for Respective Subclasses by Adjusting Channel Attenuation Statistical Distribution Parameters of Statistical Hybrid Models - Part 2: Numerical Results for the Overhead and Underground Medium-Voltage Power Grids," Trends in Renewable Energy, vol. 5, no. 3, pp. 258-281, Aug. 2019. [Online]. Available: http://futureenergysp.com/index.php/tre/article/view/100/pdf

[34] A. G. Lazaropoulos, "Virtual Indicative Broadband over Power Lines Topologies for Respective Subclasses by Adjusting Channel Attenuation Statistical Distribution Parameters of Statistical Hybrid Models - Part 3: The Case of Overhead Transmission Power Grids," Trends in Renewable Energy, vol. 5, no. 3, pp. 282-306, Aug. 2019. [Online]. Available: http://futureenergysp.com/index.php/tre/article/view/101/pdf 
[35] A. G. Lazaropoulos, "Statistical Channel Modeling of Overhead Low Voltage Broadband over Power Lines (OV LV BPL) Networks - Part 2: The Numerical Results of Class Map Footprints of Real OV LV BPL Topologies, Branch Line Faults and Hook Style Energy Thefts," Trends in Renewable Energy, vol. 6, no. 1, pp. 88-109, Mar. 2020. [Online]. Available: http://futureenergysp.com/index.php/tre/article/download/113/pdf

[36] A. G. Lazaropoulos, "Best L1 Piecewise Monotonic Data Approximation in Overhead and Underground Medium-Voltage and Low-Voltage Broadband over Power Lines Networks: Theoretical and Practical Transfer Function Determination," Hindawi Journal of Computational Engineering, vol. 2016, Article ID 6762390, 24 pages, 2016. doi:10.1155/2016/6762390. [Online]. Available: https://www.hindawi.com/journals/jcengi/2016/6762390/cta/

[37] I. C. Demetriou, "Algorithm 863: L2WPMA, a Fortran 77 package for weighted least-squares piecewise monotonic data approximation," ACM Transactions on Mathematical Software (TOMS), vol. 33, no.1, pp. 6, 2007.

[38] A. G. Lazaropoulos, "Detection of Energy Theft in Overhead Low-Voltage Power Grids - The Hook Style Energy Theft in the Smart Grid Era," Trends in Renewable Energy, vol. 5, no. 1, pp. 12-46, Oct. 2018. [Online]. Available: http://futureenergysp.com/index.php/tre/article/view/81/pdf

[39] A. G. Lazaropoulos, "Measurement Differences, Faults and Instabilities in Intelligent Energy Systems-Part 1: Identification of Overhead High-Voltage Broadband over Power Lines Network Topologies by Applying Topology Identification Methodology (TIM)," Trends in Renewable Energy, vol. 2, no. 3, pp. 85-112, Oct. 2016. [Online]. Available: http://futureenergysp.com/index.php/tre/article/view/26/32

[40] A. G. Lazaropoulos, "Main Line Fault Localization Methodology in Smart Grid Part 1: Extended TM2 Method for the Overhead Medium-Voltage Broadband over Power Lines Networks Case," Trends in Renewable Energy, vol. 3, no. 3, pp. 2-25, Dec. $2017 . \quad$ [Online]. Available: http://futureenergysp.com/index.php/tre/article/view/36

[41] A. G. Lazaropoulos, "New Coupling Schemes for Distribution Broadband over Power Lines (BPL) Networks," Progress in Electromagnetics Research B, vol. 71, pp. 39-54, 2016. [Online]. Available: http://www.jpier.org/PIERB/pierb71/02.16081503.pdf

[42] A. G. Lazaropoulos, "Power Systems Stability through Piecewise Monotonic Data Approximations - Part 1: Comparative Benchmarking of L1PMA, L2WPMA and L2CXCV in Overhead Medium-Voltage Broadband over Power Lines Networks," Trends in Renewable Energy, vol. 3, no. 1, pp. 2-32, Jan. 2017. [Online]. Available: http://futureenergysp.com/index.php/tre/article/view/29/34

[43] A. G. Lazaropoulos, "Measurement Differences, Faults and Instabilities in Intelligent Energy Systems - Part 2: Fault and Instability Prediction in Overhead High-Voltage Broadband over Power Lines Networks by Applying Fault and Instability Identification Methodology (FIIM)," Trends in Renewable Energy, vol. 2, no. 3, pp. 113-142, Oct. 2016. [Online]. Available: http://futureenergysp.com/index.php/tre/article/view/27/33

[44] A. G. Lazaropoulos, "Power Systems Stability through Piecewise Monotonic Data Approximations - Part 2: Adaptive Number of Monotonic Sections and Performance of L1PMA, L2WPMA and L2CXCV in Overhead Medium-Voltage 
Broadband over Power Lines Networks," Trends in Renewable Energy, vol. 3, no. 1, pp. 33-60, Jan. 2017. [Online]. Available: http://futureenergysp.com/index.php/tre/article/view/30/35

[45] A. G. Lazaropoulos, "Special Cases during the Detection of the Hook Style Energy Theft in Overhead Low-Voltage Power Grids through HS-DET Method - Part 1: High Measurement Differences, Very Long Hook Technique and "Smart" Hooks," Trends in Renewable Energy, vol. 5, no. 1, pp. 60-89, Jan. 2019. [Online]. Available: http://futureenergysp.com/index.php/tre/article/view/82/pdf

[46] A. G. Lazaropoulos, "Special Cases during the Detection of the Hook Style Energy Theft in Overhead Low-Voltage Power Grids through HS-DET Method - Part 2: Different Measurement Differences, Feint "Smart" Hooks and Hook Interconnection Issues," Trends in Renewable Energy, vol. 5, no. 1, pp. 90-116, Jan. 2019. [Online]. Available: http://futureenergysp.com/index.php/tre/article/view/83/pdf

[47] I. C. Demetriou, "A Decomposition Theorem for the Least Squares Piecewise Monotonic Data Approximation Problem," Springer Approximation and Optimization, pp. 119-134, 2019.

[48] I. C. Demetriou and M. J. D. Powell, "Least squares smoothing of univariate data to achieve piecewise monotonicity", IMA Journal of Numerical Analysis, vol. 11, no. 3, pp. 411-432, 1991.

[49] I. C. Demetriou, "An application of best $L 1$ piecewise monotonic data approximation to signal restoration," IAENG International Journal of Applied Mathematics, vol. 53, no. 4, pp. 226-232, 2013.

[50] I. C. Demetriou, "L1PMA: A Fortran 77 Package for Best L1 Piecewise Monotonic Data Smoothing," Computer Physics Communications, vol. 151, no. 1, pp. 315-338, 2003.

[51] I. C. Demetriou, "L2CXCV: A Fortran 77 package for least squares convex/concave data smoothing," Computer physics communications, vol. 174, no.8, pp. 643-668, 2006.

[52] http://cpc.cs.qub.ac.uk/summaries/ADRF

[53] A. G. Lazaropoulos, "Business Analytics and IT in Smart Grid - Part 3: New Application Aspect and the Quantitative Mitigation Analysis of Piecewise Monotonic Data Approximations on the iSHM Class Map Footprints of Overhead Low-Voltage Broadband over Power Lines Topologies Contaminated by Measurement Differences," Trends in Renewable Energy, vol. 6, no. 2, pp. 204223, May 2020.

Article copyright: (C) 2020 Athanasios G. Lazaropoulos. This is an open access article distributed under the terms of the Creative Commons Attribution 4.0 International License, which permits unrestricted use and distribution provided the original author and source are credited. 